\title{
26. GEOCHEMISTRY OF BASALTS: DEEP SEA DRILLING PROJECT SITES 482, 483, AND 485 NEAR THE TAMAYO FRACTURE ZONE, GULF OF CALIFORNIA ${ }^{1}$
}

\author{
M. F. J. Flower, Department of Geological Sciences, University of Illinois, Chicago, Illinois \\ R. G. Pritchard, Institut für Mineralogie der Ruhr-Universität, 463 Bochum-Querenburg, \\ Federal Republic of Germany, and Philips GmbH, 3500 Kassel, Miramstrasse 87, Federal Republic of Germany \\ H.-U. Schmincke, Institut für Mineralogie der Ruhr-Universität, 463 Bochum-Querenburg, \\ Federal Republic of Germany \\ and \\ Paul T. Robinson, Department of Geology, Dalhousie University, Halifax, Nova Scotia B3H 3J5, Canada
}

\section{INTRODUCTION}

Recent investigations of the southern Gulf of California $\left(22^{\circ} \mathrm{N}\right)$ on Leg 65 of the Deep Sea Drilling Project (DSDP) (Fig. 1) allow important comparisons with drilled sections of ocean crust formed at different spreading rates. During Leg 65 the Glomar Challenger drilled seven basement holes at sites forming a transect across the ridge axis near the Tamayo Fracture Zone (Fig. 1). An additional site was drilled on the fracture zone itself, where a small magnetic "diapir" was located. Together with the material from Site 474 (drilled during Leg 64) the cores recovered at these sites are representative of the upper basaltic and sedimentary crust formed since the initial opening of the Gulf. The pattern of magmatic accretion at the ridge axis is conditioned by the moderate to high rate of spreading $(\sim 6 \mathrm{~cm} / \mathrm{y}$.) and comparatively high sedimentation rates that now characterize the Gulf of California. In terms of spreading rate, this region is intermediate between the "superfast" East $\mathrm{Pa}$ cific Rise axis to the south (up to $17 \mathrm{~cm} / \mathrm{y}$.) and the slow-spreading Mid-Atlantic Ridge $(2-4 \mathrm{~cm} / \mathrm{y}$.) both of which have been extensively studied by dredging and drilling.

In this chapter, we present data on major and trace elements for drilled basaltic rocks from the Tamayo transect. The samples were taken during shipboard investigation of the core and are representative of the igneous rocks encountered at each site.

\section{CRUSTAL CONSTRUCTION IN THE GULF OF CALIFORNIA}

The Gulf of California represents an early stage in the formation of an ocean basin and includes within its confines an active accretion zone, numerous transform fractures, and passive continental margins (Fig. 1). Spreading began in the Gulf about 3.5 m.y. ago, and today reflects a distinct transition in the spreading styles observed along the East Pacific Rise. South of the Tamayo Fracture Zone the rise shows comparatively sim-

\footnotetext{
${ }^{1}$ Lewis, B. T. R., Robinson, P., et al., Init. Repts. DSDP, 65: Washington (U.S. Govt. Printing Office).
}

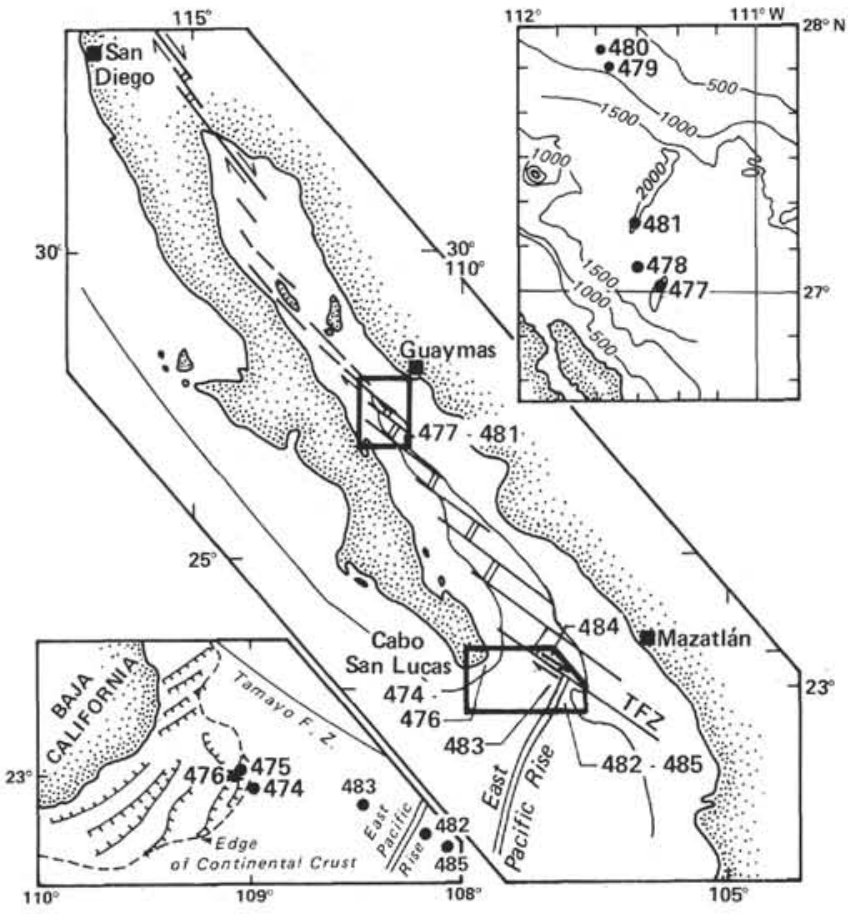

Figure 1. Map of the Gulf of California, showing areas studied on DSDP Legs 64 and 65 . Note change from a dilational regime to a dominantly strike-slip regime at the Tamayo Fracture Zone (TFZ).

ple dilation. To the north, strike-slip motion is increasingly dominant as transform fractures become more common.

The southern part of the Gulf of California has been studied in considerable detail by a variety of techniques. The Leg 65 transect across the ridge axis spans most of its temporal evolution, while the "RITA" Project used submersibles to obtain morphologic, petrologic, and geochemical data for the ridge axis at $21^{\circ} \mathrm{N}$ (CYAMEX, in press) and along the Tamayo Fracture Zone (Kastens et al., 1979). Results of the "ROSE" experiment (e.g., Reid et al., 1977) yielded evidence for intermittent melt zones beneath the ridge axis. Seismic reflection profiling across the axis at $21-23^{\circ} \mathrm{N}$ (Lewis, 1979) suggests that accretion at contiguous ridge segments is episodic and may reflect flow of magma along the axis. 
Studies of the Mid-Atlantic Ridge suggest that crustal accretion at slow-spreading axes is also strongly episodic and generally accompanied by tectonic disruption. The resulting crustal structure is apparently composed of imbricated lenses with little lateral continuity. Seafloor topography in the Gulf of California appears to consist of linear basalt ridges, parallel to the main axis, separated by broad flat-floored valleys. The ridges appear to comprise pillow basalt complexes, while the valleys are floored (or partially filled by) thick massive flows (Francheteau et al., 1979). There is little evidence of tectonic disturbance. The ridges may represent waning stages of a constructive cycle, while the massive units appear to represent earlier, more prolific phases of such cycles. The crustal sections revealed by drilling show an analogous pattern to that reported from submersible studies. At the deepest site, pillow lavas interlayered with thin massive flows are found in the lower levels of the section, but the upper levels consist of thick massive units of more magnesian character.

The general characteristics of crust generated at the mouth of the Gulf of California are depicted in Figure 2 for the Leg 65 sections. Sedimentation rates were lower at Site 483 west of the ridge axis than at the other sites (Rangin, this volume). Thus an equivalent depth of basement penetration at this site corresponds to a more extensive igneous section than at the other sites. The lower sections at Site 483 reveal interlayered pillows and thin massive flows. This type of sequence was not encountered at Sites 482 and 485 , where only thick massive units were observed.

Excellent intrasite correlation was established at Sites 482 and 483 , reflecting lithologic and chemical continuity of emplacement units over distances of at least 2,000 meters. No evidence for tectonic disturbance of the crust is apparent.

\section{SAMPLING AND ANALYTICAL TECHNIQUES}

The shipboard studies established criteria for the lithologic subdivision of the basalts recovered. Petrography, frequency of chill margins, and sedimentary intercalations in the drilled sections allowed inference of "cooling unit" divisions (not synonymous with "eruptive" units) which range from single pillows a few centimeters thick to massive units some tens of meters thick. The cooling units identified are summarized and related to lithologic units in Appendixes A and B. The cooling units are taken as a basic reference for integrating chemical and other data since it may be assumed that the properties of any given unit share a common origin. Lithologic "subunits" were defined in some cases to allow for unrecovered cooling unit boundaries.

In general, glass cooling selvedges are associated with pillows and thin massive flows. Most of the glass samples examined were from the lower parts of Holes 483 and $483 \mathrm{~B}$, although a small amount was sampled from Holes 482B, D, and F. Glass was not observed at Sites 484 and 485 . However, aphyric, nonpillowed basalt units are common at all sites and may be considered equivalent to liquid compositions. Thus, basalt liquid-fraction compositions may be studied at all of the sites and compared with the compositions of phyric basalts from the same sites. Data for mineral compositions (Viereck, personal communication) indicate that phenocrysts mostly represent equilibrium assemblages grown in situ rather than accumulated phases from previous, or spatially separated, crystallization episodes. However, there is evidence for some phenocryst accumulation and resorption.

Major element concentrations were determined by X-ray fluorescence on samples prepared as glass beads. Lithium tetraborate and metaborate (rock to flux ratio $1: 4$ ) were used for fusion, and analyses were made using a Philips PW $1450 \mathrm{X}$-ray fluorescence spectrometer.
Analysis for $\mathrm{Fe}^{2+}$ was conducted by digesting samples in hydrofluoric acid and silver perchlorate solution followed by potentiometric titration of excess perchlorate with standard potassium bromide solution. $\mathrm{H}_{2} \mathrm{O}$ was determined by ignition in a dry inert gas atmosphere followed by coulometric titration of the effluent gas in barium perchlorate solution. Samples 482C-12-1, 92-118; 483B-8-3, 2-20; 483B$28-1,42-59$; and $485 \mathrm{~A}-25-1,117-143$ were analyzed as interlaboratory comparisons; data are given in Table 1 with analytical statistics.

\section{MAJOR ELEMENT VARIATION}

The complete data set comprises 187 analyses of basalt sampled from Sites 482,483 , and 485 . The major element analyses are given in Table 2 and averaged by chemical type in Table 3, while the trace elements are given in Table 4 and their ratios in Table 5. Most samples are from massive or pillowed flow units; they are fine grained with holocrystalline to intersertal textures and range from moderately phyric to aphyric. Glass selvedges associated with thin flows at Site 482 and with pillow lavas at Site 483 were sampled and analyzed using an electron microprobe. These data are given by Flower and O'Hearn (this volume) with additional whole-rock analyses. Together, the data are used to study compositional variations in the magmatic liquid fraction and the total eruptive magma (liquid plus any crystalline fraction) during the formation of crust at these localities. Following preliminary shipboard studies, we defined "chemical types" that correspond to distinct compositional-stratigraphic groupings of the data. These form a basis for interpreting petrogenetic processes.

Slight to moderate degrees of alteration were encountered in the core, as reflected by the presence of smectite-and in rare cases, talc-the latter indicating moderately high temperatures. However, altered zones were carefully avoided in sampling for this study. "Fresh" rock analyses (i.e., not significantly leached of $\mathrm{Ca}^{2+}$ and $\mathrm{Mg}^{2+}$ ) were corrected for interstitial and vesicular calcite by removing $\mathrm{CO}_{2}$-equivalent $\mathrm{CaO}$ and normalizing to dry weight. Using corrected data sets for each site, the chemical types were established graphically in relation to the stratigraphic and lithologic associations recorded on shipboard. With few exceptions, the chemical types defined on shipboard remain unchanged in light of the expanded data sets obtained in this study. The stratigraphic extent of lavas composing a single chemical type often correspond to rock sequences built up during a single eruption or eruptive phase. Chemical types thus appear to be a useful indicator of the chronologic patterns of fractionation.

Previous studies of oceanic basement (e.g., Flower et al., 1977; Byerly and Wright, 1978; Flower and Robinson, 1981a and b; Bryan et al., 1978) suggest that compositional differences between chemical types may reflect the effects of both deep-seated and comparatively shallow-level fractionation. Such processes would be expected to include fractional crystallization, as constrained by polybaric phase equilibria and mixing of magma batches at various stages of the liquids' evolution. Although we assume that lavas belonging to a single chemical type reflect a common fractionation and eruptive history, we focus attention on variations both within and between each type. The average composi- 
tions of the chemical types encountered at Leg 65 sites are given in Table 3 , and the petrography of the core material is summarized in Appendix B in relation to chemical types and lithologic units.

Compositional variations are depicted according to chemical type in plots of weight percent $\mathrm{FeO}^{*}$ (i.e., total Fe oxide), $\mathrm{Al}_{2} \mathrm{O}_{3}$, and $\mathrm{TiO}_{2}$ versus $\mathrm{MgO}$. This type of oxide variation diagram permits simple graphical distinction between liquid variation trends resulting from fractional crystallization and contrasting trends resulting from the effects of phenocryst accumulation and, possibly, alteration.

\section{Whole-Rock Variation, Site $\mathbf{4 8 2}$}

The Site 482 chemical types (A-H inclusive) are best represented at Hole 482B and less completely at Holes $482 \mathrm{C}$, D, and F. All but one of the cooling units identified at this site are massive and probably eruptive, while the chemical types may include more than a single cooling unit. The chemical variation (Fig. 3) appears to reflect a combination of liquid phase variation and phenocryst accumulation processes. Chemical Type $\mathrm{A}$ is represented by the uppermost basalt unit in Holes 482B, C, and D (Lithologic Unit 1), an aphyric flow extending at least 1000 meters laterally. Chemical Type $\mathrm{E}$ is slightly more $\mathrm{Al}_{2} \mathrm{O}_{3}$-rich, but otherwise very similar to Type $\mathrm{A}$, and they are grouped together in the diagrams. Chemical Types $\mathrm{B}$ and $\mathrm{F}$ are indistinguishable in terms of major elements, but Type $\mathrm{F}$ is characterized by a notably higher $\mathrm{Ni}$ content and a lower $\mathrm{Zr} / \mathrm{Ni}$ ratio than $\mathrm{B}$ (Table 5). Chemical Type $F$ is absent from Hole $482 C$ but separates Types A and B in Hole 482B. Both Types B and F are distinguished from Type $\mathrm{A}$ by lower $\mathrm{TiO}_{2}$ and $\mathrm{FeO}^{*}$ and higher $\mathrm{Al}_{2} \mathrm{O}_{3}$ for equivalent $\mathrm{MgO}$ content (Fig. 3). Chemical Type D is represented at the base of Hole 482B (only) by phyric Lithologic Units 7 and 8 and also by the intercalated Unit 5 . The plagioclase-phyric lithology and chemical variation of Chemical Type D suggest a derivation from a low- $\mathrm{TiO}_{2}$ magma (Chemical Type A?) involving some accumulation of plagioclase (Fig. 3). In general, there is excellent correlation between the holes at this site for Chemical Types A and B and, between Holes 482B and D for Type F. It is assumed that Type D would be encountered by deeper penetration in Holes $482 \mathrm{C}$ and D. Least-squares analysis of the major element variation within and between the Site 482 chemical types (Table 6) suggests that two or more stages of fractionation were involved, both before and after segregation of the magma into the batches now represented by chemical types.

\section{Whole-Rock Variation, Site $\mathbf{4 8 3}$}

The patterns of whole-rock variation observed at Site 483 (Fig. 4) show that the chemical types encountered are related to the inferred emplacement mode, namely massive versus pillowed cooling units. On the basis of lithologic and magnetic stratigraphy (Day, this volume), drilling rate, and geophysical logging (see site summaries, and Salisbury, this volume), we have established an excellent cross-hole correlation between cooling units and chemical types. The upper four basalt units show a simple cross-hole correspondence matched by thick sedimentary interlayers. Lithologic Unit 5 at Hole 483 (Chemical Type E) is equivalent to a nonrecovered basalt interval intercalated with the sediment in Hole 483B. Likewise, a nonrecovered basalt interval recorded by logging in Hole 483 corresponds to a pillow sequence (Chemical Type I) in Hole 483B. The cross-hole correlation continues down-section, with several massive flow units of Chemical Type $\mathrm{H}$ traced between each hole. The resultant stratigraphic synthesis allows complete identification of the massive units at this site.

In summary, the crustal structure at Site 483 may be divided into two parts:

1) Chemical Types A-D (Lithologic Units 1-4) make up the "upper" massive units, separated by thick sediment intercalations. These units are comparatively rich in $\mathrm{MgO}\left(7.2-10.0\right.$ wt. \%), $\mathrm{CaO}$, and $\mathrm{Al}_{2} \mathrm{O}_{3}$, and low in $\mathrm{TiO}_{2}, \mathrm{FeO}$, and $\mathrm{P}_{2} \mathrm{O}_{5}$.

2) Chemical types E-M (Lithologic Units 5-9) make up an intercalated series of pillow and thin massive basalt sequences. These have $\mathrm{MgO}$ contents of 7.0-7.4 wt. $\%$ and distinctly higher $\mathrm{TiO}_{2}$ and $\mathrm{FeO} *$ values than do the "upper" massive units.

Several discrete massive units belonging to Chemical Types $\mathrm{H}$ and $\mathrm{K}$ show similar variation patterns (Fig. 4). These units are notably more fractionated than the "upper" massive units. These groups depart compositionally from the overall variation trend represented by the whole-rock data and, more particularly, the glass data (Flower and O'Hearn, this volume). Least-squares analysis of the intraunit variation suggests that complex phenocryst redistribution processes involving the accumulation of plagioclase took place in some cases.

The pillow units (Chemical Types F, I, J, L, and M) are generally more phyric than the massive units. While showing similar individual trends, these units reflect considerably more diffuse variation than the massive units. Least-squares tests indicate that simple phenocryst removal during cotectic fractionation is insufficient to explain the variations observed between and within chemical types (Table 6). Chemical Types D, B, $\mathrm{K}$, and $\mathrm{H}$ appear to require some accumulation of plagioclase concomitant with the removal of olivine and clinopyroxene to satisfy internal mass balance requirements. This appears to be a common characteristic of the massive eruptions but is not supported by the sparsely phyric or aphyric petrography of these units.

\section{Whole-Rock Variation, Site $\mathbf{4 8 5}$}

Shipboard analysis of samples from Site 485 was not completed on board. Thus chemical types were not previously defined for this site. In this study, chemical types were defined which in several cases correspond to single massive units. For the eight basaltic units drilled at Site 485, chemical types were assigned as follows: $1: \mathrm{A}, \mathrm{B}$, $\mathrm{C}$, and E; $2: \mathrm{Z}$ ?; 3 (not analyzed); 4: H; 5: I; 6: J; 7: K; and $8:$ L. Each lithologic unit probably represents a single emplacement event. However, in some cases (e.g., Units 1 and 5 especially) each may comprise several intrusive and/or extrusive subunits. This is reflected by 


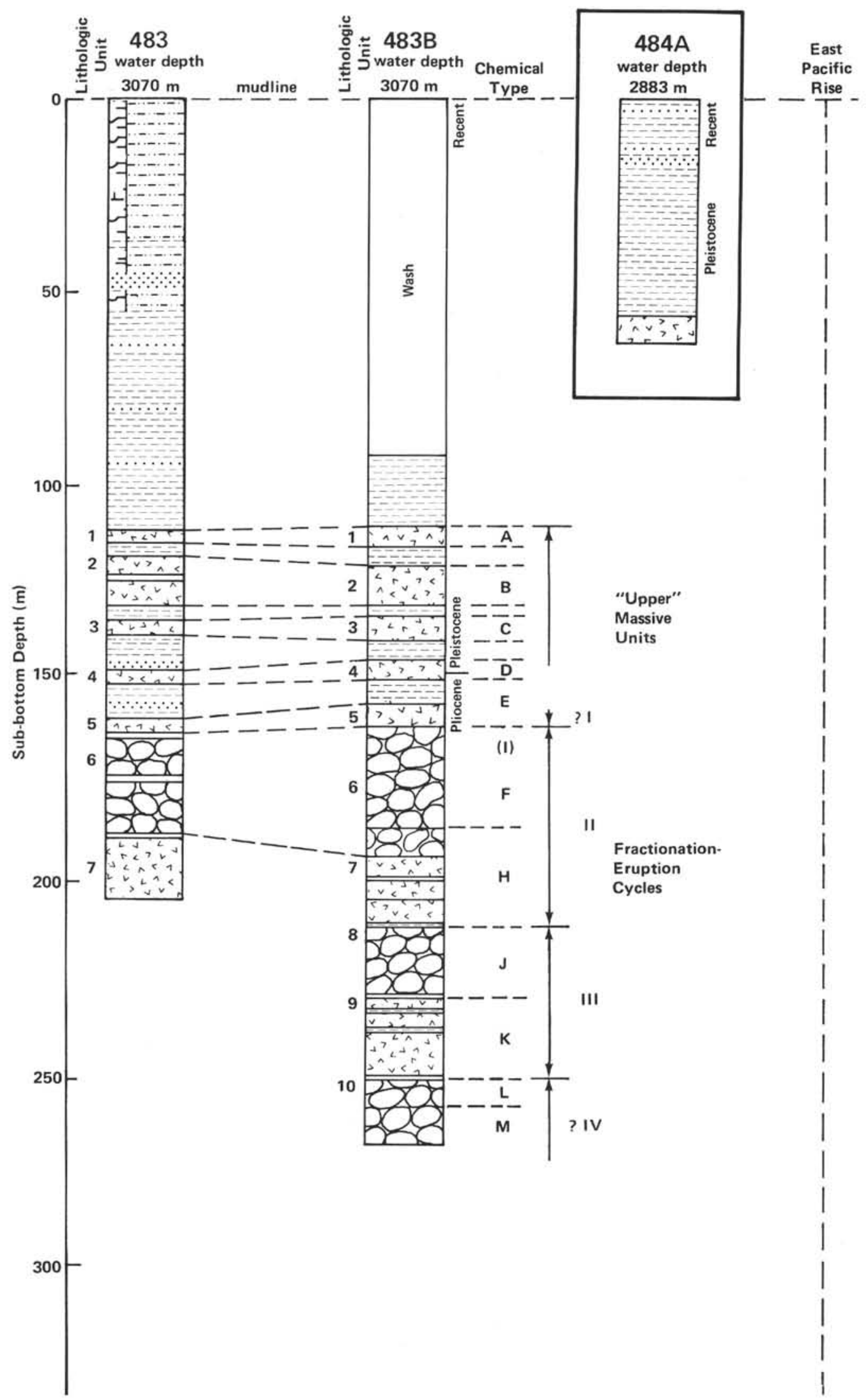

Figure 2. Sections drilled during Leg 65. Chemical types identified in this study and between-hole correlations at Sites 482 and 483 are indicated. No samples from Site 484 were analyzed. (Water depths shown in meters at the top of each section.) 


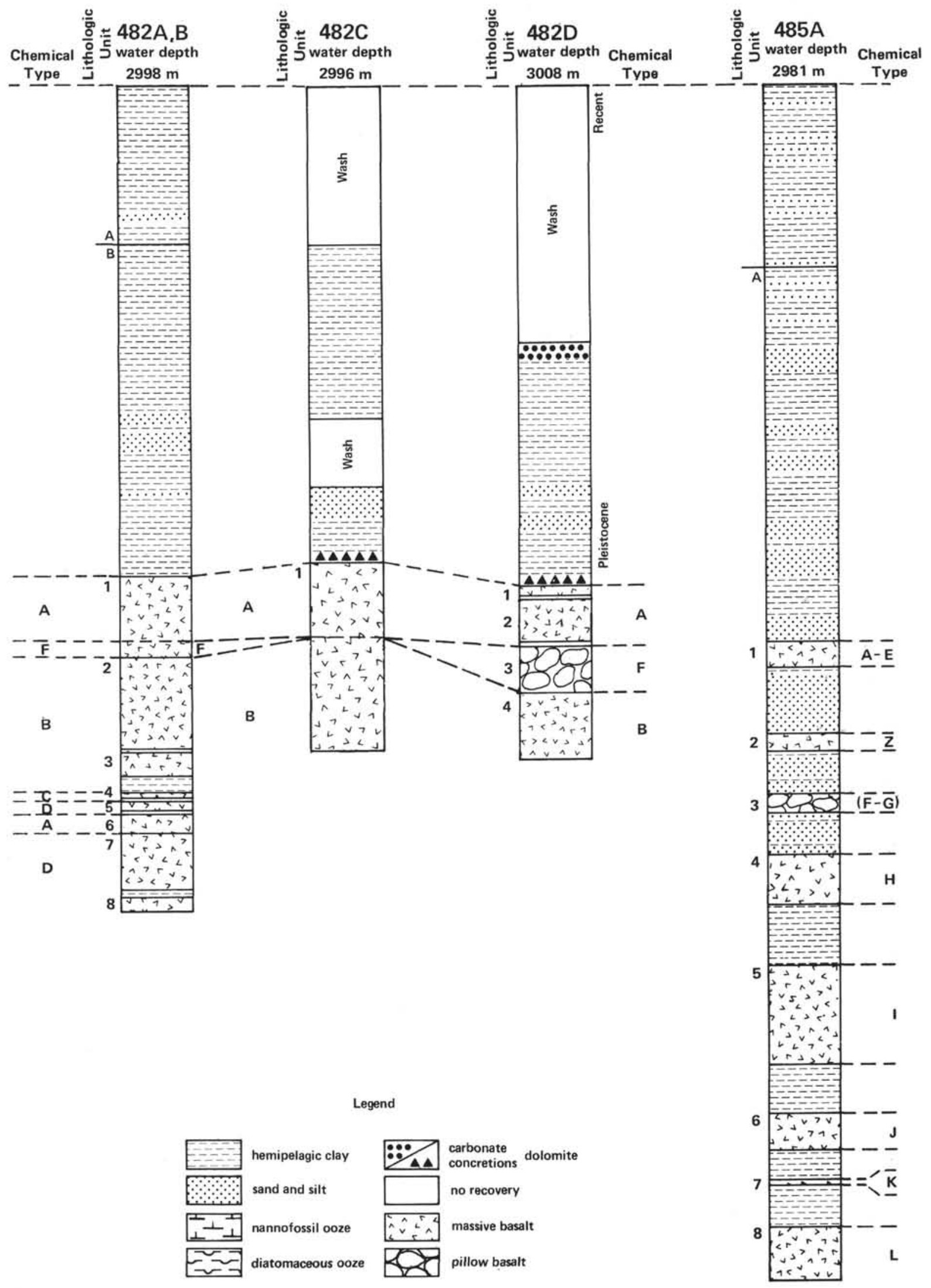

Figure 2. (Continued). 
Table 1. Interlaboratory comparison of analyses.

\begin{tabular}{|c|c|c|c|c|c|c|c|}
\hline \multicolumn{8}{|c|}{ Sample $482 \mathrm{C}-12-1,91-118 \mathrm{~cm}$} \\
\hline Oxide & $\mathrm{ALB}^{\mathrm{a}}$ & $\mathrm{BIR}^{\mathrm{a}}$ & BOC $^{\mathrm{a}}$ & $\mathrm{BRE}^{\mathrm{a}}$ & $I C L^{a}$ & Mean & $2 a$ \\
\hline $\mathrm{SiO}_{2}$ & 50.21 & 50.11 & 50.30 & 50.24 & 50.29 & 50.22 & 0.07 \\
\hline $\mathrm{Al}_{2} \mathrm{O}_{3}$ & 14.74 & 14.94 & 14.60 & 14.78 & 14.64 & 14.74 & 0.13 \\
\hline $\mathrm{Fe}_{2} \mathrm{O}_{3}{ }^{*}$ & 11.12 & 11.11 & 11.00 & 11.29 & 11.02 & 11.11 & 0.11 \\
\hline $\mathrm{MgO}$ & 7.78 & 7.98 & 7.81 & 7.88 & 7.79 & 7.85 & 0.09 \\
\hline $\mathrm{CaO}$ & 11.78 & 12.08 & 12.40 & 12.26 & 12.10 & 12.13 & 0.23 \\
\hline $\mathrm{Na}_{2} \mathrm{O}$ & 2.47 & 2.27 & 2.47 & 1.96 & 2.38 & 2.31 & 0.21 \\
\hline $\mathrm{K}_{2} \mathrm{O}$ & 0.06 & 0.06 & 0.05 & 0.07 & 0.06 & 0.06 & $<0.01$ \\
\hline $\mathrm{TiO}_{2}$ & 1.23 & 1.30 & 1.27 & 1.29 & 1.26 & 1.27 & 0.03 \\
\hline $\mathrm{P}_{2} \mathrm{O}_{5}$ & 0.10 & 0.11 & 0.11 & 0.15 & 0.12 & 0.12 & 0.02 \\
\hline $\mathrm{MnO}$ & 0.16 & 0.15 & 0.18 & 0.18 & 0.16 & 0.17 & 0.01 \\
\hline Total & 99.65 & 100.14 & 100.20 & 100.10 & 99.82 & 99,98 & \\
\hline $\begin{array}{l}\mathrm{H}_{2} \mathrm{O}^{+} \\
\mathrm{CO}_{2}\end{array}$ & $\begin{array}{l}0.90 \\
\text { nd }\end{array}$ & $\begin{array}{l}\text { nd } \\
\text { nd }\end{array}$ & $\begin{array}{l}0.93 \\
0.13\end{array}$ & $\begin{array}{l}\text { nd } \\
\text { nd }\end{array}$ & $\begin{array}{l}\text { nd } \\
\text { nd }\end{array}$ & & \\
\hline \multicolumn{8}{|c|}{ Sample 483B-8-3, 2-20 cm } \\
\hline Oxide & ALB & BIR & BOC & BRE & ICL & Mean & $2 \sigma$ \\
\hline $\mathrm{SiO}_{2}$ & 48.59 & 48.90 & 48.50 & 48.33 & 48.80 & 48.63 & 0.23 \\
\hline $\mathrm{Al}_{2} \mathrm{O}_{3}$ & 16.66 & 16.42 & 16.40 & 16.52 & 16.58 & 16.52 & 0.18 \\
\hline $\mathrm{Fe}_{2} \mathrm{O}_{3} *$ & 10.06 & 9.64 & 9.57 & 9.86 & 9.84 & 9.79 & 0.19 \\
\hline $\mathrm{MgO}$ & 9.05 & 9.76 & 9.29 & 9.35 & 9.26 & 9.34 & 0.26 \\
\hline $\mathrm{CaO}$ & 12.89 & 12.05 & 12.20 & 12.28 & 12.21 & 12.12 & 0.15 \\
\hline $\mathrm{Na}_{2} \mathrm{O}$ & 2.27 & 2.14 & 2.20 & 2.27 & 2.13 & 2.20 & 0.07 \\
\hline $\mathrm{K}_{2} \mathrm{O}$ & 0.04 & 0.05 & 0.04 & 0.04 & 0. & 0.04 & $<0.01$ \\
\hline $\mathrm{TiO}_{2}$ & 1.01 & 1.07 & 1.06 & 1.07 & 1.06 & 1.05 & 0.03 \\
\hline $\mathrm{P}_{2} \mathrm{O}_{5}$ & 0.07 & 0.08 & 0.08 & 0.12 & 0.09 & 0.09 & $<0.02$ \\
\hline MnO & 0.15 & 0.15 & 0.16 & 0.16 & 0.17 & 0.16 & $<0.01$ \\
\hline Total & 99.79 & 100.26 & 99.49 & 100.00 & 100.19 & 99.95 & \\
\hline $\begin{array}{l}\mathrm{H}_{2} \mathrm{O}^{+} \\
\mathrm{CO}_{2}\end{array}$ & $\begin{array}{l}1.94 \\
\text { nd }\end{array}$ & $\begin{array}{l}\text { nd } \\
\text { nd }\end{array}$ & $\begin{array}{l}1.80 \\
0.35\end{array}$ & $\begin{array}{l}\text { nd } \\
\text { nd }\end{array}$ & $\begin{array}{l}\text { nd } \\
\text { nd }\end{array}$ & & \\
\hline \multicolumn{8}{|c|}{ Sample 483B-28-1, 42-59 cm } \\
\hline Oxide & ALB & BIR & $\mathrm{BOC}$ & BRE & ICL & Mean & $2 \sigma$ \\
\hline $\begin{array}{l}\mathrm{SiO}_{2} \\
\mathrm{Al}_{2} \mathrm{O}_{3}\end{array}$ & $\begin{array}{l}49.63 \\
14.35\end{array}$ & $\begin{array}{l}49.63 \\
14.48\end{array}$ & $\begin{array}{l}49.70 \\
14.10\end{array}$ & $\begin{array}{l}49.58 \\
14.17\end{array}$ & $\begin{array}{l}49.51 \\
14.17\end{array}$ & $\begin{array}{l}49.61 \\
14.26\end{array}$ & $\begin{array}{l}0.07 \\
0.15\end{array}$ \\
\hline $\mathrm{Fe}_{2} \mathrm{O}_{3} *$ & 12.40 & 12.17 & 12.00 & 12.36 & 12.03 & 12.18 & 0.19 \\
\hline $\mathrm{MgC}$ & 7.56 & & 7.72 & 7.73 & & 7.67 & 0.07 \\
\hline $\mathrm{CaO}$ & 11.19 & 11 & 11.70 & 11.66 & & 11.49 & 0.20 \\
\hline $\mathrm{Na}_{2} \mathrm{O}$ & & & 2.66 & & & 2.54 & 0.19 \\
\hline $\mathrm{K}_{2} \mathrm{O}$ & 0. & & & & & & $<0.01$ \\
\hline $\mathrm{TiO}_{2}$ & 1.72 & 1.92 & 1.77 & 1.81 & 1. & 1.8 & 0.08 \\
\hline $\mathrm{P}_{2} \mathrm{O}_{5}$ & 0.15 & & 0.15 & 0.19 & & 0.1 & 0.02 \\
\hline $\mathrm{MnO}$ & 0.18 & 0.16 & 0.19 & 0.18 & 0.22 & 0.19 & 0.02 \\
\hline Total & 100.00 & 100.26 & 100.10 & 100.01 & 99.63 & 99.99 & \\
\hline $\mathrm{H}_{2} \mathrm{O}^{+}$ & 1.18 & nd & 1.25 & nd & nd & & \\
\hline $\mathrm{CO}_{2}$ & & & 0.19 & , nat & & & \\
\hline \multicolumn{8}{|c|}{ Sample $485 \mathrm{~A}-25-1,117-143 \mathrm{~cm}$} \\
\hline Oxide & ALB & BIR & $\mathrm{BOC}$ & BRE & ICL & Mean & $2 \sigma$ \\
\hline & 49.69 & 49.34 & 49.80 & 49.09 & 49.20 & 49.44 & 0.32 \\
\hline $\mathrm{Al}_{2} \mathrm{O}_{3}$ & 14.61 & 14.80 & 14.70 & 14.43 & 14.53 & 14.60 & 0.14 \\
\hline $\mathrm{Fe}_{2} \mathrm{O}_{3}{ }^{*}$ & 12.44 & 12.61 & 12.60 & 12.88 & 12.60 & 12.63 & 0.16 \\
\hline $\mathrm{Mg}$ & 7.18 & 7.20 & 7.25 & 7.11 & & 7.19 & 0.05 \\
\hline $\mathrm{CaO}$ & 10.71 & 11.03 & 11.20 & 11.20 & 11.07 & 11.05 & 0.20 \\
\hline $\mathrm{Na}_{2} \mathrm{O}$ & 2.65 & & 2. & & & 2.55 & 0.07 \\
\hline $\mathrm{K}_{2} \mathrm{O}$ & 0.09 & 0.08 & 0.08 & 0.08 & 0. & 0.08 & $<0.01$ \\
\hline $\mathrm{TiO}_{2}$ & 2.0 & 2.34 & 2.13 & 2.15 & 2.1 & 2.16 & 0.10 \\
\hline $\mathrm{P}_{2} \mathrm{O}_{5}$ & 0.19 & 0.21 & 0.20 & 0.21 & 0.21 & 0.20 & $<0.01$ \\
\hline Total & 99.83 & 100.34 & 100.80 & 99.85 & 99.74 & 100.11 & \\
\hline $\mathrm{H}_{2} \mathrm{O}^{+}$ & 1.05 & nd & 1.02 & nd & nd & & \\
\hline & na & nd & 0.15 & nd & nd & & \\
\hline
\end{tabular}

${ }^{\text {a }}$ BOC-Ruhr-Univ., Bochum; BIR = Univ, of Birmingham; $\mathrm{ALB}=$ Univ. of New Mexico, Albuquerque; $\mathrm{BRE}=\mathrm{CNEXO}$, Brest; $\mathrm{ICL}=1$ mperial College, London.

significant internal chemical diversity. We infer intrusive origins where the basalt groundmass textures are particularly coarse grained. Intrusive contacts were only observed, however, for Unit 5.
In general, and in contrast to Site 483 , the chemical variation within and between the Site 485 chemical types (Fig. 5) appears consistent with a single parental magma type (cf. Site 483). However, internal variation within, for example, Type I (Unit 5) indicates a complex fractionation history prior to or during emplacement. Intraunit variation is in most cases attributed to fractional crystallization and possibly to some minor flow differentiation during emplacement. The massive units include a total of 28 lithologic subunits defined during shipboard core description.

Lithologic Unit 1 comprises Cooling Units 1-5 (A-E in Fig. 5), which correspond to at least three internal variation trends: A, E, and B-C. The upper part of Unit 1 (Type A) shows a comparatively high $\mathrm{MgO}$ content. The most magnesian sample analyzed is unaltered, shows little evidence of olivine accumulation, and appears to represent a liquid composition. The internal trends are similar to the massive unit trends at Site 483 (Fig. 4) and in some cases (see Table 6) require accumulation of plagioclase concomitant with mafic phase fractionation (Table 6). The parent batches may belong to one or several fractionation trends of a more deep-seated origin. Lithologic Unit 2 is represented by one analysis which is compositionally distinct from Chemical Types A-E. Lithologic Unit 4, in contrast, is comparatively uniform in composition (Type $\mathrm{H}$ in Fig. 5). Lithologic Unit 5 is similar to Unit 1 and shows considerable internal variation. This unit, identified on shipboard as a single cooling unit (9), can be subdivided chemically into at least five stratigraphically contiguous units, each corresponding to one or several lithologic subunit divisions (i.e., Subunits 9/16-9/22). Such complex variation suggests that the emplacement of this unit may have involved a combination of extrusion and late-stage intrusive events. Least-squares solutions for the internal variation indicate that similar phenocryst redistribution patterns affected several compositionally distinct parent batches. As for Site 483 Chemical Type D, the very sparsely phyric character of Unit 5 does not suggest plagioclase accumulation, although this appears to be required by the least-squares solutions (Table 6). A more complex origin is thus indicated. Lithologic Units 6, 7, and 8 consist of sparsely- to moderately-phyric basalt and each represents a single cooling unit. The data are insufficient to categorize Unit 7 (the single analysis, Type K in Fig. 5, is strongly indicative of plagioclase accumulation), but Units 6 and 8 ( $\mathrm{J}$ and $\mathrm{L}$ in Fig. 5) reflect little internal variation compared to Units 5 and 1 . They are also indistinguishable from each other, but separated stratigraphically by the distinctive Type $\mathrm{K}$.

Of the eight major basalt units cored at Site 485 , at least two (1 and 5) show complex internal variation patterns. These are indicative of discrete fractionation events superimposed on one or more compositional trends of deeper-seated origin.

\section{TRACE ELEMENT VARIATION}

The trace elements $\mathrm{Cr}, \mathrm{Ni}, \mathrm{Cu}, \mathrm{Zn}, \mathrm{Rb}, \mathrm{Sr}, \mathrm{Y}, \mathrm{Zr}$, and $\mathrm{Nb}$ were analyzed for all 187 samples (Tables 4 and 5) 
Table 2. Major element compositions of fresh basalts from DSDP Sites 482,483 , and $485^{\mathrm{a}}$.

\begin{tabular}{|c|c|c|c|c|c|c|c|c|c|c|}
\hline $\begin{array}{l}\text { Sample } \\
\text { (interval in } \mathrm{cm} \text { ) }\end{array}$ & $\mathrm{SiO}_{2}$ & $\mathrm{Al}_{2} \mathrm{O}_{3}$ & $\mathrm{FeO} \cdot$ & $\mathrm{MgO}$ & $\underset{\text { (wt. }}{\mathrm{CaO}}$ & $\mathrm{Na}_{2} \mathrm{O}$ & $\mathrm{K}_{2} \mathrm{O}$ & $\mathrm{TiO}_{2}$ & $\mathrm{P}_{2} \mathrm{O}_{5}$ & $\mathrm{MnO}$ \\
\hline \multicolumn{11}{|l|}{ Hole $482 \mathrm{~B}$} \\
\hline 1. 99-104 & 50.73 & 14 & 10.39 & 8.10 & 11.53 & 2.57 & .06 & 1.80 & 15 & 19 \\
\hline & 50 & & & $\pi$ & & & & & 11 & \\
\hline $2-13$ & $\begin{array}{l}50.27 \\
50.28\end{array}$ & $\begin{array}{l}15 . \\
15 . \\
\text {. }\end{array}$ & $\begin{array}{l}10.05 \\
10.03\end{array}$ & $\begin{array}{l}7.73 \\
8.04\end{array}$ & 12.15 & $\begin{array}{l}2.62 \\
2,37\end{array}$ & 15 & $\begin{array}{l}1.39 \\
1.42\end{array}$ & 12 & \\
\hline $\begin{array}{l}14-3,2-6 \\
14-20\end{array}$ & $\begin{array}{l}50.28 \\
50.21\end{array}$ & $\begin{array}{l}15.01 \\
14.85\end{array}$ & $\begin{array}{l}10.03 \\
10.22\end{array}$ & $\begin{array}{l}8.04 \\
8.00\end{array}$ & $\begin{array}{l}11.99 \\
12.14\end{array}$ & $\begin{array}{l}2.37 \\
2.45\end{array}$ & 07 & $\begin{array}{l}1.42 \\
1.43\end{array}$ & $\begin{array}{l}0.11 \\
0.12\end{array}$ & \\
\hline $15-1,12$ & 50.49 & & & 7.96 & 11.44 & 2.51 & & 1.48 & 0.11 & \\
\hline & 51.04 & & & 8.13 & & & & & & \\
\hline$-3,14$ & 50.20 & & & $\begin{array}{r}7.33 \\
7.45\end{array}$ & & & & & & \\
\hline $\begin{array}{l}-3,1,14 \\
-2,19\end{array}$ & $\begin{array}{l}50.49 \\
50.78\end{array}$ & $\begin{array}{l}14.69 \\
14.72\end{array}$ & $\begin{array}{l}10.32 \\
10.28\end{array}$ & $\begin{array}{r}7.45 \\
7.46\end{array}$ & 12.33 & $\begin{array}{r}2.50 \\
249\end{array}$ & 03 & $\begin{array}{l}1.51 \\
1.51\end{array}$ & 13 & \\
\hline & & & $\begin{array}{r}9.28 \\
9.89\end{array}$ & $\begin{array}{l}7.46 \\
7.26\end{array}$ & 12.06 & 2.49 & & 1.31 & 0.13 & 0.18 \\
\hline & & & 0 & 7.31 & & 2.7. & & 1.56 & 14 & 0.19 \\
\hline & so. & 15 & 9.58 & 7.6 & & & & & & \\
\hline 22 & & 15. & 9.69 & 7.84 & & 2.52 & (1) & 1.50 & 0.14 & \\
\hline $24-3,110-115$ & 50.57 & 14.21 & 10.58 & 7.53 & 11.67 & 2.78 & 0.06 & 1.87 & 0.16 & 0.17 \\
\hline
\end{tabular}
Hole $482 \mathrm{C}$

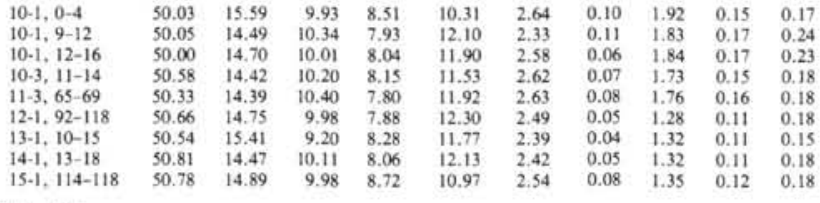
Hole 482D

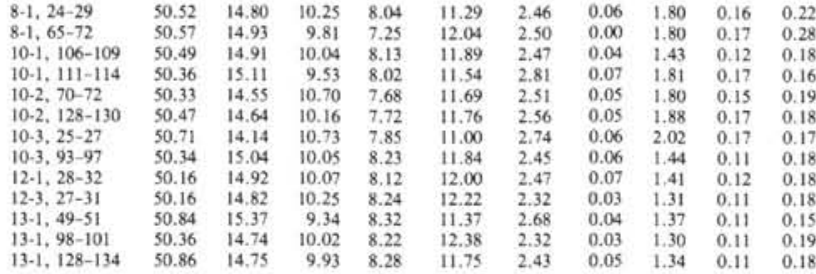
Hole $482 \mathrm{~F}$

$\begin{array}{lllllllllll}5-1,133-139 & 50.57 & 14.34 & 11.35 & 6.84 & 10.97 & 2.85 & 0.11 & 2.14 & 0.20 & 0.19 \\ 11-1,125-128 & 50.39 & 14.91 & 10.02 & 8.28 & 11.86 & 2.45 & 0.05 & 1.43 & 0.11 & 0.18\end{array}$ Hole 483

$\begin{array}{lllllllllll}14,1,58-68 & 50.88 & 15.34 & 9.27 & 8.03 & 11.89 & 2.66 & 0.06 & 1.23 & 0.09 & 0.17\end{array}$ $\begin{array}{lrlrllllllll}13-1,93-97 & 50.89 & 15.24 & 8.89 & 8.77 & 12.71 & 2.59 & 0.06 & 1.24 & 0.09 & 0.16 \\ 16-2,64-70 & 50.77 & 14.71 & 10.02 & 7.90 & 11.81 & 2.75 & 0.07 & 1.41 & 0.10 & 0.18\end{array}$ $\begin{array}{lllllllllll}16-2,99-100 & 50.55 & 14.84 & 9.71 & 7.89 & 12.10 & 2.75 & 0.07 & 1.42 & 0.11 & 0.16 \\ 16-3.26 & 50.68 & 15.04 & 9.49 & 8.04 & 11.98 & 2.77 & 0.07 & 1.34 & 0.10 & 0.17\end{array}$ $\begin{array}{lllllllllll}16-3,2-6 & 50.68 & 15.04 & 9.49 & 8.04 & 11.98 & 2.77 & 0.07 & 1.34 & 0.10 & 0.17\end{array}$ $\begin{array}{lllllllllll}172,17-17 & 50.00 & 17.07 & 7.87 & 8.82 & 12.54 & 2.22 & 0.03 & 1.00 & 0.07 & 0.15\end{array}$

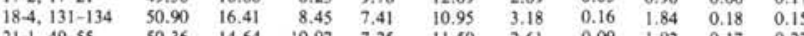
$\begin{array}{lllllllllll}21-1,49-55 & 50.36 & 14.64 & 10.97 & 7.25 & 11.50 & 2.61 & 0.09 & 1.92 & 0.17 & 0.22\end{array}$ $\begin{array}{lllllllllll}21-1,57-61 & 50.42 & 14.93 & 10.81 & 7.12 & 11.54 & 2.49 & 0.12 & 1.90 & 0.17 & 0.21 \\ 21-3,6-9 & 49.96 & 14.80 & 10.84 & 7.44 & 11.42 & 2.73 & 0.09 & 1.96 & 0.18 & 0.24\end{array}$ $\begin{array}{lllrrrrrrrr}21-3.6-9 & 49.96 & 14.80 & 10.84 & 7.44 & 11.42 & 2.73 & 0.09 & 1.96 & 0.18 & 0.24 \\ 22-2,68-72 & 50.04 & 15.48 & 9.95 & 7.20 & 12.31 & 2.58 & 0.08 & 1.67 & 0.15 & 0.21\end{array}$ $\begin{array}{lllllllllll}22.2 .745 & 50.26 & 15.27 & 10.13 & 7.43 & 12.03 & 2.48 & 0.08 & 1.68 & 0.14 & 0.20\end{array}$

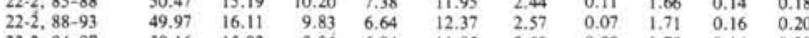
$\begin{array}{lllllllllll}22-2,94-99 & 50.16 & 15.92 & 9.96 & 6.94 & 11.95 & 2.60 & 0.09 & 1.70 & 0.14 & 0.20\end{array}$ $\begin{array}{lllllllllll}23-1,10-16 & 49.76 & 15.90 & 10.08 & 6.91 & 12.28 & 2.52 & 0.05 & 1.74 & 0.16 & 0.20 \\ 2.1 .52-57 & 49.76 & 15.17 & 10.94 & 6.96 & 1.79 & 3.06 & 0.08 & 2.35 & 0.22 & 0.17\end{array}$

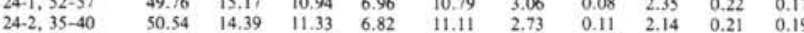

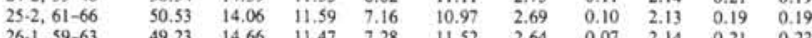

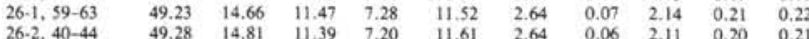

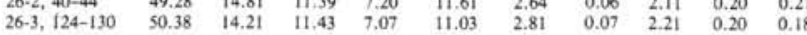
Hole 483B

\begin{tabular}{|c|c|c|c|c|c|c|c|c|c|}
\hline $26-30$ & 50.77 & 15.00 & 9.24 & 8.24 & 12.20 & 2.63 & 0.07 & 1.24 & 0.09 \\
\hline & $\begin{array}{r}50.44 \\
50.37\end{array}$ & 14.59 & 10.15 & 7.68 & $\begin{array}{l}12.30 \\
\end{array}$ & 2.73 & & 1.42 & 0.10 \\
\hline $\begin{array}{r}26-31 \\
66-61\end{array}$ & $\begin{array}{l}50.37 \\
50.67\end{array}$ & $\begin{array}{l}14.54 \\
14.54\end{array}$ & 10.16 & 7.94 & 12,33 & 2.66 & 0.05 & 1.36 & 0.10 \\
\hline $\begin{array}{l}5-661 \\
2-6\end{array}$ & $\begin{array}{l}.66 \\
80\end{array}$ & $\begin{array}{r}14.54 \\
14.76\end{array}$ & $\begin{array}{r}9.91 \\
10.08\end{array}$ & $\begin{array}{l}7.98 \\
7.41\end{array}$ & $\begin{array}{l}12.19 \\
12.16\end{array}$ & $\begin{array}{l}2.67 \\
2.75\end{array}$ & $\begin{array}{l}0.05 \\
0.05\end{array}$ & $\begin{array}{l}1.37 \\
\\
\end{array}$ & 0.10 \\
\hline $8-1,29-35$ & .26 & $\begin{array}{l}\begin{array}{l}44.76 \\
17.42\end{array}\end{array}$ & $\begin{array}{r}10.08 \\
8.00\end{array}$ & $\begin{array}{l}7.41 \\
7.76\end{array}$ & $\begin{array}{l}12.16 \\
12.63\end{array}$ & $\begin{array}{l}2.75 \\
2.40\end{array}$ & $\begin{array}{l}0.05 \\
0.04\end{array}$ & $\begin{array}{l}1.38 \\
1.01\end{array}$ & $\begin{array}{l}0.09 \\
0.07\end{array}$ \\
\hline $55-61$ & 49.39 & 16.43 & 8.53 & 9.94 & 12.30 & 2.13 & 0.03 & 0.98 & 0.07 \\
\hline 12 & & 16.83 & 8.14 & 8.91 & 12.81 & 2.28 & & & \\
\hline 26 & & & $\begin{array}{l}8.26 \\
8.25\end{array}$ & 8.86 & & & & 1.10 & \\
\hline $\begin{array}{c}2-2 \\
92\end{array}$ & $\begin{array}{l}49.32 \\
49.75\end{array}$ & $\begin{array}{l}16.72 \\
15.60\end{array}$ & $\begin{array}{r}8.75 \\
1030\end{array}$ & $\begin{array}{l}9.44 \\
7.18\end{array}$ & 11.92 & 2.24 & & 1.07 & 0.08 \\
\hline $38-44$ & 50.49 & $\begin{array}{l}13.60 \\
14.65\end{array}$ & $\begin{array}{l}10.30 \\
10.95\end{array}$ & $\begin{array}{l}7.18 \\
7.07\end{array}$ & $\begin{array}{l}12.00 \\
11.54\end{array}$ & & & $\begin{array}{l}1.57 \\
1.89\end{array}$ & \\
\hline $1,60-64$ & 50.18 & 14.65 & 10.83 & 7.21 & 11.58 & & 0 & 1.93 & 0.17 \\
\hline 4 & & & 10.61 & 7.12 & 11.74 & & & 1.95 & \\
\hline 67 & & 14.83 & II. & 7.16 & & & & 95 & \\
\hline $\begin{array}{l}66- \\
74\end{array}$ & $\begin{array}{r}50.200 \\
50.36\end{array}$ & $\begin{array}{l}14.68 \\
14.66\end{array}$ & $\begin{array}{l}11.20 \\
10.98\end{array}$ & 6.98 & $\begin{array}{l}11.57 \\
11.67\end{array}$ & & & 1.94 & 0.18 \\
\hline 92 & $\begin{array}{r}50.360 \\
50.26\end{array}$ & $\begin{array}{l}44.66 \\
14.82\end{array}$ & 10.88 & 7.23 & & & & $\begin{array}{r}1.92 \\
1.76\end{array}$ & \\
\hline 10 & & 14.78 & 11.01 & 6.87 & & & & 1.86 & \\
\hline & & 14.52 & 11.50 & 7.06 & & & & & \\
\hline 11 & s & 14.99 & & 7.18 & & & & 89 & 18 \\
\hline 1,12 & 0.05 & $\begin{array}{l}44.99 \\
1480\end{array}$ & 10. & 7.14 & ${ }_{3}^{8}$ & & & & \\
\hline .124 & & $\begin{array}{l}14.80 \\
14.82\end{array}$ & & 67 & & & & & \\
\hline 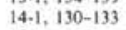 & & & & 7.10 & & & & $\begin{array}{l}1.00 \\
1.78\end{array}$ & \\
\hline & & 14.30 & & & & & & 26 & 20 \\
\hline & & 1440 & $4 x$ & 41 & & & & 17 & 19 \\
\hline $.18-22$ & 0.61 & 14.10 & 11.25 & & 10.40 & & & 2.20 & 0.20 \\
\hline
\end{tabular}

Table 2. (Continued).

\begin{tabular}{|c|c|c|c|c|c|c|c|c|c|c|}
\hline $\begin{array}{l}\text { Sample } \\
\text { (interval in } \mathrm{cm} \text { ) }\end{array}$ & $\mathrm{SiO}_{2}$ & $\mathrm{Al}_{2} \mathrm{O}_{3}$ & $\mathrm{FeO}^{*}$ & $\mathrm{MgO}$ & $\underset{\text { (wt }}{\mathrm{CaO}}$ & $\mathrm{Na}_{2} \mathrm{O}$ & $\mathrm{K}_{2} \mathrm{O}$ & $\mathrm{TiO}_{2}$ & $\mathrm{P}_{2} \mathrm{O}_{5}$ & $\mathrm{MnO}$ \\
\hline Hole 482B & & & & & & & & & & \\
\hline $9-2,17-21$ & 50.23 & 14.24 & 11.51 & 6.77 & 11.15 & 2.71 & 0.10 & 2.15 & 0.21 & 0.19 \\
\hline & 50.22 & & 11.36 & & 10.78 & 20 & 0.08 & 2.29 & 0.24 & 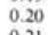 \\
\hline 9 & 40 & & & 7 & 14 & & 11 & & & \\
\hline & 49.81 & & & & & 2.82 & .00 & & & 0.19 \\
\hline 2.9 & & & 11.00 & 7.40 & $\begin{array}{l}06 \\
22\end{array}$ & $\begin{array}{l}2.81 \\
2.73\end{array}$ & $\begin{array}{l}12 \\
11\end{array}$ & ${ }_{09}^{13}$ & 20 & 19 \\
\hline 2,1 & 50.41 & 14.11 & 11.55 & 7,16 & .13 & 2,68 & 10 & 2.08 & 20 & 0.20 \\
\hline & & & & & & 2.6 & & 215 & 19 & \\
\hline & & & & & & & 10 & & & 20 \\
\hline & $\begin{array}{r}49.83 \\
4977-1\end{array}$ & & & 7 & & & 11 & & & 17 \\
\hline -2., 5 & & & & $\begin{array}{l}7,62 \\
7.33\end{array}$ & $4_{2}^{4}$ & $\begin{array}{l}2.79 \\
2.79\end{array}$ & 10 & 19 & 20 & $\begin{array}{l}19 \\
19\end{array}$ \\
\hline $\begin{array}{l}20-2,20-1929 \\
20-2,146\end{array}$ & $\begin{array}{l}59.84 \\
49.84\end{array}$ & $\begin{array}{l}1.54 \\
14.83\end{array}$ & $\begin{array}{l}11.31 \\
10.84\end{array}$ & 7.30 & .32 & $\begin{array}{l}2.79 \\
2.92\end{array}$ & ${ }_{10}^{11}$ & $\begin{array}{l}2.22 \\
2.05\end{array}$ & 20 & 18 \\
\hline $21-2,57-61$ & & & & 7.4 & 57 & 2.75 & 11 & 1.8 & 16 & \\
\hline & & & $\begin{array}{l}10.59 \\
0.57\end{array}$ & & & & & & & 22 \\
\hline $\begin{array}{l}-2,63 \\
-2,71\end{array}$ & & & $\begin{array}{r}9.57 \\
0.45\end{array}$ & 7 & & 2.63 & 07 & & & \\
\hline $\begin{array}{l}-2,7 \\
4,1\end{array}$ & & & $\begin{array}{l}10.45 \\
10.22\end{array}$ & $\begin{array}{l}7.72 \\
7.48\end{array}$ & $\begin{array}{l}97 \\
87\end{array}$ & 2.5 & 05 & 59 & 4 & \\
\hline $25-2$, & 66.93 & 16. & 3.61 & 7.12 & 1.65 & 2.40 & 07 & 0.63 & 20 & 0.03 \\
\hline $25 \cdot 2$ & & & 10.36 & 7,4 & & & & & 17 & \\
\hline & & & & & & & & & & 20 \\
\hline & & & & & & & & & & 21 \\
\hline 1 & & & $\begin{array}{r}11.10 \\
9.63\end{array}$ & & $\begin{array}{l}96 \\
46\end{array}$ & $\begin{array}{l}2.68 \\
2.91\end{array}$ & $4_{7}^{4}$ & $\begin{array}{ll}75 \\
88\end{array}$ & & \\
\hline-1.11 & $\begin{array}{l}50.32 \\
50.49\end{array}$ & 14.73 & $\begin{array}{l}9.033 \\
10.79\end{array}$ & 7.06 & 48 & 2.75 & 08 & 85 & 16 & \\
\hline & 50.36 & & & & & & & & & \\
\hline & & & & & & & & & & \\
\hline & & & & & & & & & & \\
\hline${ }_{23}^{25}$ & $\begin{array}{l}50 . \\
50\end{array}$ & 14 & & & $\begin{array}{l}57 \\
03\end{array}$ & 2 & & & & \\
\hline $28 \cdot 2,28-$ & $\begin{array}{l}50.25 \\
50.26\end{array}$ & $\begin{array}{l}14.43 \\
14.40\end{array}$ & $\begin{array}{l}10.36 \\
10.36\end{array}$ & $\begin{array}{l}8.80 \\
8.00\end{array}$ & .04 & 2. & 07 & 65 & 15 & \\
\hline $28-3,37-$ & & 14 & & & & & & & & \\
\hline & & & & & & & & & & \\
\hline & & & & & & & & & & \\
\hline$\frac{5}{7}$ & & & & & & & & & & \\
\hline & & & & & & & & & & \\
\hline $29-1,57$ & & 14.73 & 10.71 & 0 & 98 & 2 & & & & \\
\hline & & & & & & & & & & \\
\hline & & & & & & & & & & \\
\hline & & & & & & & & & & \\
\hline & & & 10.06 & 7. & & & & & & \\
\hline 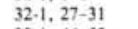 & & tat & 9,47 & & & & & & & \\
\hline & & & & & & & & & & \\
\hline & & & & & & & & & & \\
\hline & & & & & & & & & & \\
\hline $327,04-60$ & & & & & & & 11 & & & 0.23 \\
\hline ole $483 \mathrm{C}$ & & & & & & & & & & \\
\hline $4-4,58-66$ & 50.69 & 14.66 & 9.96 & 7.72 & 2.33 & 2.64 & 0.05 & 1.33 & 0.10 & 0.17 \\
\hline Hole $485 \mathrm{~A}$ & & & & & & & & & & \\
\hline & & & & & & & & & & \\
\hline & & & & & & & & & & \\
\hline & & & 9.36 & & & & & & & \\
\hline & & 88 & 11.89 & & & & & & & \\
\hline & & & & & & & & & & \\
\hline & & & & & & & & & & \\
\hline & & & & & & & & & & \\
\hline & & & & & & & & & & \\
\hline 2 & 50 & & & & & & & & & \\
\hline & & & & & & & & & & \\
\hline & & & & & & & & & & \\
\hline & & & & & & & & & & \\
\hline & & & & & & & & & & \\
\hline & & & & & & & & & & \\
\hline & & & & & & & & & & \\
\hline & & & & & & & & & & \\
\hline & & & & & & & & & & \\
\hline & & & & & & & & & & \\
\hline & & & & & & & & & & \\
\hline & & & & & & & & & & \\
\hline & & & & & & & & & & \\
\hline & & & & & & & & & & \\
\hline & & & & & & & & & & \\
\hline & & & & & & & & & & \\
\hline & & & & & & & & & & \\
\hline & & & & & & & & & & \\
\hline & & & & & & & & & & \\
\hline & & & & & & & & & & \\
\hline & & & & & & & & & & \\
\hline & & & & & & & & & & \\
\hline & & & & & & & & & & \\
\hline & & & & & & & & & & \\
\hline & & & & & & & & & & \\
\hline & & & & & & & & & & \\
\hline & & & & & & & & & & \\
\hline & & & & & & & & & & \\
\hline & & & & & & & & & & \\
\hline & & & & & & & & & & \\
\hline & & & & & & & & & & \\
\hline & & & & & & & & & & \\
\hline & & & & & & & & & & \\
\hline & & & & & & & & & 0.16 & 0.18 \\
\hline
\end{tabular}

a Values in wt. $\%_{0 ;}$ normalized and corrected for carbonate contamination. 
Table 3. Average compositions of basalt chemical types at DSDP Sites 482,483 , and $485 .^{\mathrm{a}}$

\begin{tabular}{|c|c|c|c|c|c|c|c|c|c|c|}
\hline $\mathrm{SiO}_{2}$ & $\mathrm{Al}_{2} \mathrm{O}_{3}$ & $\mathrm{FeO}^{*}$ & $\mathrm{MgO}$ & $\begin{array}{l}\mathrm{CaO} \\
\text { (wt. \% }\end{array}$ & $\mathrm{Na}_{2} \mathrm{O}$ & $\mathrm{K}_{2} \mathrm{O}$ & $\mathrm{TiO}_{2}$ & $\mathrm{P}_{2} \mathrm{O}_{5}$ & $\mathrm{MnO}$ & $\begin{array}{c}\text { Chemical } \\
\text { Type }\end{array}$ \\
\hline \multicolumn{11}{|l|}{ Site 482} \\
\hline 50.58 & 14.71 & 10.25 & 7.96 & 11.63 & 2.58 & 0.09 & 1.82 & 0.16 & 0.21 & A \\
\hline 50.47 & 15.14 & 10.12 & 8.04 & 11.95 & 2.48 & 0.06 & 1.44 & 0.12 & 0.18 & F \\
\hline 50.85 & 14.94 & 9.94 & 8.20 & 11.93 & 2.46 & 0.04 & 1.35 & 0.11 & 0,18 & B \\
\hline 51.20 & 14.70 & 10.03 & 8.16 & 12.00 & 2.30 & 0.06 & 1.28 & 0.11 & 0.17 & C \\
\hline 50.35 & 15.54 & 9.88 & 7.42 & 12.25 & 2.65 & 0.04 & 1.56 & 0.14 & 0.18 & D \\
\hline 50.67 & 14.67 & 10.32 & 7.85 & 11.54 & 2.67 & 0.06 & 1.89 & 0.17 & 0.18 & E \\
\hline 50.79 & 14.40 & 11.40 & 6.87 & 11.02 & 2.86 & 0.11 & 2.15 & 0.20 & 0.19 & G \\
\hline 50.55 & 14.96 & 10.05 & 8.31 & 11.90 & 2.46 & 0.05 & 1.43 & 0.11 & 0.18 & H \\
\hline \multicolumn{11}{|l|}{ Site 483} \\
\hline 51.04 & 15.25 & 9.17 & 8.04 & 12.31 & 2.64 & 0.06 & 1.24 & 0.09 & 0.16 & B \\
\hline 50.78 & 14.77 & 9.97 & 7.86 & 12.17 & 2.73 & 0.06 & 1.39 & 0.10 & 0.17 & C \\
\hline 49.84 & 16.87 & 8.27 & 9.09 & 12.38 & 2.27 & 0.04 & 1.01 & 0.07 & 0.16 & D \\
\hline 51.09 & 16.47 & 8.48 & 7.44 & 10.99 & 3.19 & 0.16 & 1.85 & 0.18 & 0.15 & E \\
\hline 50.06 & 15.70 & 10.36 & 7.22 & 12.07 & 2.62 & 0.06 & 1.58 & 0.13 & 0.20 & 1 \\
\hline 50.34 & 15.08 & 10.72 & 7.14 & 11.80 & 2.61 & 0.09 & 1.86 & 0.17 & 0.20 & F \\
\hline 50.33 & 14.54 & 11.41 & 7.28 & 10.97 & 2.80 & 0.10 & 2.18 & 0.21 & 0.19 & $\mathrm{H}$ \\
\hline 50.40 & 15.04 & 10.25 & 7.56 & 11.93 & 2.65 & 0.08 & 1.73 & 0.15 & 0.21 & J \\
\hline 50.36 & 14.67 & 10.71 & 7.67 & 11.63 & 2.72 & 0.09 & 1.82 & 0.16 & 0.19 & K \\
\hline 50.42 & 14.91 & 10.52 & 7.60 & 11.60 & 2.65 & 0.11 & 1.81 & 0.16 & 0.23 & L \\
\hline 50,42 & 15.59 & 9.63 & 7.64 & 11.94 & 2.68 & 0.12 & 1.62 & 0.15 & 0.20 & M \\
\hline 50.87 & 14.71 & 9.99 & 7.75 & 12.37 & 2.65 & 0.05 & 1.33 & 0.10 & 0.17 & Z \\
\hline \multicolumn{11}{|l|}{ Site 485} \\
\hline 50.00 & 17.36 & 9.37 & 7.13 & 11.25 & 3.08 & 0.03 & 1.54 & 0.10 & 0.13 & A \\
\hline 50.50 & 13.93 & 11.93 & 6.83 & 11.62 & 2.60 & 0.09 & 2.09 & 0.19 & 0.23 & B \\
\hline 50.62 & 13.83 & 12.11 & 6.89 & 11.38 & 2.56 & 0.07 & 2.12 & 0.19 & 0.23 & C \\
\hline 50.83 & 14.18 & 11.72 & 7.12 & 11.11 & 2.54 & 0.06 & 2.04 & 0.19 & 0.21 & E \\
\hline 49.97 & 15,47 & 12.09 & 7.85 & 8.87 & 2.98 & 0.05 & 2.33 & 0.20 & 0.19 & z \\
\hline 50.26 & 14.83 & 11.40 & 7.07 & 10.76 & 2.93 & 0.14 & 2.24 & 0.20 & 0.17 & H \\
\hline 50.10 & 14.71 & 11.07 & 7.82 & 11.12 & 2.62 & 0.13 & 2.05 & 0.18 & 0.19 & 1 \\
\hline 50.68 & 14.59 & 10.68 & 7.78 & 11.48 & 2.60 & 0.06 & 1.79 & 0.15 & 0.18 & $\mathrm{~J}$ \\
\hline 49.95 & 19.61 & 9.85 & 6.41 & 7.55 & 3.68 & 0.06 & 2.48 & 0.21 & 0.19 & K \\
\hline 50.44 & 15.00 & 10.74 & 7.68 & 11.27 & 2.58 & 0.06 & 1.87 & 0.16 & 0.19 & L \\
\hline
\end{tabular}

a Values in wr.\%, normalized and corrected for carbonate.

and the data used to refine the conclusions based on the major element variations. In general, the chemical types discussed above are also consistent with the trace element data. Incompatible (or 'low- $\mathrm{K}_{\mathrm{D}}$ ') element enrichment factors are moreover consistent with the fractionation models based on major element mass balance considerations (Table 6). $\mathrm{Rb}$ and $\mathrm{Nb}$ values are generally below or close to detection limits and are therefore unreliable as petrogenetic indicators. The remaining elements reflect a wide range of solid-liquid partitioning behavior, from highly "compatible" to incompatible types. $\mathrm{Zr}$, and to a lesser extent $\mathrm{Y}$, are effectively excluded from olivine, plagioclase, and clinopyroxene during their crystallization in a tholeiite liquid. $\mathrm{Sr}$ is partitioned into plagioclase but not olivine or clinopyroxene and is thus a sensitive monitor for plagioclase fractionation. $\mathrm{Cu}$ and $\mathrm{Zn}$ do not discriminate significantly between the silicate phases and in general show compatible behavior. They are, however, very sensitive to sulfide fractionation. $\mathrm{Ni}$ and $\mathrm{Cr}$ are excluded from plagioclase but partition strongly, although differentially, into olivine and clinopyroxene. Taken together, the trace elements may be used to constrain models developed earlier from major element variation patterns.

As a general observation it is noted that the $\mathrm{Zr} / \mathrm{Ti}$ ratios are not constant for all chemical types at any one site and appear to distinguish broader groupings of these types. The $\mathrm{Zr} / \mathrm{Y}$ ratios also vary between types for equivalent values of $\mathrm{Zr}$ and reflect a significant positive correlation with $\mathrm{Zr}$ content. $\mathrm{Zr} / \mathrm{Y}$ ratios of about 2.4, corresponding to $50-60 \mathrm{ppm} \mathrm{Zr}$, increase to about 3.1 for $140-150 \mathrm{ppm} \mathrm{Zr}$. These observations are important in evaluating the relative significance of fractional crys-

Table 4. Trace element abundances in fresh basalts from DSDP Sites 482,483 , and 485 .

\begin{tabular}{cccccccccc}
\hline $\begin{array}{c}\text { Sample } \\
\text { (interval in } \mathrm{cm})\end{array}$ & $\mathrm{Cu}$ & $\mathrm{Zn}$ & $\mathrm{Rb}$ & $\begin{array}{c}\mathrm{Nb} \\
\begin{array}{c}\mathrm{Cr} \\
(\mathrm{ppm})\end{array}\end{array}$ & $\mathrm{Ni}$ & $\mathrm{Sr}$ & $\mathrm{Y}$ & $\mathrm{Zr}$ \\
\hline
\end{tabular}

Hole 482B

13-1, 99-104 $14-1,91-94$

$14-1,132-137$

$14-3,2-6$

$14-4,16-20$

$15-1,12-16$

$16-4,100-104$

20-1, 27-30

20-3, 144-147

21-2, 19-23

21-3, 58-63

22-2, 67-72

22-2, 130-136

22-3, 103-107

22-4, 97-102

$24-3,110-115$

Hole $482 \mathrm{C}$

$\begin{array}{lll}56 & 85 & 4 \\ 84 & 84 & 2 \\ 72 & 76 & 1 \\ 76 & 82 & 2 \\ 70 & 90 & 2 \\ 68 & 78 & 3 \\ 65 & 74 & 4 \\ 92 & 93 & 3 \\ 72 & 87 & 1 \\ 80 & 78 & 5 \\ 79 & 86 & 1 \\ 82 & 89 & 2 \\ 71 & 83 & 0 \\ 66 & 80 & 1 \\ 82 & 85 & 1 \\ 68 & 87 & 7\end{array}$

$\begin{array}{ll}7 & 272 \\ 8 & 254 \\ 9 & 240 \\ 8 & 230 \\ 8 & 240 \\ 9 & 236 \\ 6 & 180 \\ 0 & 202 \\ 8 & 201 \\ 8 & 193 \\ 8 & 231 \\ 8 & 242 \\ 6 & 226 \\ 8 & 222 \\ 8 & 244 \\ 8 & 191\end{array}$

$\begin{array}{rrrr}70 & 107 & 39 & 113 \\ 102 & 91 & 35 & 86 \\ 90 & 97 & 32 & 83 \\ 90 & 92 & 33 & 84 \\ 83 & 93 & 33 & 84 \\ 91 & 92 & 34 & 86 \\ 57 & 87 & 31 & 75 \\ 76 & 116 & 35 & 100 \\ 68 & 112 & 34 & 96 \\ 56 & 104 & 30 & 80 \\ 58 & 124 & 36 & 107 \\ 62 & 123 & 35 & 106 \\ 57 & 124 & 35 & 106 \\ 71 & 120 & 35 & 102 \\ 67 & 122 & 35 & 105 \\ 63 & 110 & 41 & 123\end{array}$

Hole 482D

$$
\begin{aligned}
& 10-1,0-4 \\
& 10-1,9-12 \\
& 10-1,12-16 \\
& 10-3,11-14 \\
& 11-3,65-69 \\
& 12-1,92-118 \\
& 13-1,10-15 \\
& 14-1,13-18
\end{aligned}
$$

$15-1,114-118$

8-1, 24-29

8-1, 65-72

$10-1,106-109$

10-1, 111-114

$10-2,70-72$

$10-2,128-130$

$10-3,25-27$

10-3, 93-97

$12-1,28-32$

$12-3,27-31$

13-1, 49-51

13-1, 98-101

$13-1,128-134$

$\begin{array}{ll}71 & 89 \\ 79 & 90 \\ 78 & 105 \\ 75 & 78 \\ 94 & 97 \\ 72 & 70 \\ 89 & 83 \\ 70 & 79 \\ 80 & 8\end{array}$

89
90
105
78
97
70
83
79
85

6
3
0
7
1
1
3
3
1

7

260
275
278
285
295
185
176
166
188

260
275
278
285
295
185
176
166
188

$\begin{array}{rrrr}61 & 111 & 39 & 123 \\ 67 & 106 & 41 & 119 \\ 64 & 107 & 41 & 118 \\ 73 & 104 & 37 & 111 \\ 71 & 104 & 38 & 113 \\ 57 & 89 & 32 & 77 \\ 58 & 93 & 31 & 77 \\ 54 & 90 & 32 & 76 \\ 55 & 97 & 32 & 79\end{array}$

Hole $482 \mathrm{~F}$

5-1, 133-139

$11-1,125-128$

70
77
70
78
71
88
67
87
92
78
78
102
81

82

94

84

90

89

92
87

83

91
80

80

90

$\begin{array}{rr}8 & 2 \\ 8 & 296 \\ 9 & 228 \\ 7 & 299 \\ 8 & 24 \\ 9 & 263 \\ 8 & 206 \\ 10 & 235 \\ 8 & 2 \\ 7 & 192 \\ 8 & 185 \\ 9 & 1 \\ 7 & 1\end{array}$

264
296
228
299
249
263
206
235
238
192
185
191
179

$\begin{array}{rrrr}71 & 106 & 40 & 116 \\ 69 & 111 & 39 & 116 \\ 91 & 92 & 33 & 84 \\ 73 & 111 & 39 & 116 \\ 66 & 106 & 40 & 115 \\ 66 & 108 & 40 & 121 \\ 63 & 107 & 43 & 127 \\ 93 & 92 & 33 & 85 \\ 90 & 91 & 33 & 84 \\ 55 & 90 & 32 & 77 \\ 56 & 98 & 32 & 80 \\ 57 & 92 & 32 & 78 \\ 61 & 92 & 32 & 79\end{array}$

Hole 483

14-1, 58-68

$15-1$,

16-2, 64-70

$16-2,99-100$

$16-3,2-6$

$17-2,13-17$

$17-2,17-21$

$18-4,131-134$

21-1, 49-55

21-1, 57-61

$21-3,6-9$

22-2, 68-72

22-2, 74-77

22-2, 85-88

22-2, 88-93

22-2, 94-99

23-1, 10-16

24-1, 52-57

24-2, 35-40

25-2, 61-66

$$
\begin{array}{rrrrrrrrr}
95 & 97 & 2 & 9 & 163 & 64 & 104 & 40 & 117 \\
93 & 87 & 5 & 8 & 288 & 93 & 89 & 33 & 84
\end{array}
$$

$\begin{array}{lllllllll}93 & 87 & 5 & 8 & 288 & 93 & 89 & 33 & 84\end{array}$

$\begin{array}{rrrrrrrrr}99 & 73 & 3 & 8 & 363 & 62 & 117 & 28 & 74 \\ 91 & 65 & 2 & 8 & 348 & 65 & 114 & 28 & 73 \\ 81 & 84 & 1 & 8 & 230 & 52 & 94 & 32 & 77 \\ 91 & 82 & 4 & 8 & 235 & 54 & 98 & 30 & 77 \\ 67 & 73 & 0 & 8 & 279 & 56 & 96 & 30 & 73 \\ 96 & 62 & 1 & 8 & 315 & 108 & 99 & 25 & 59 \\ 88 & 67 & 2 & 7 & 360 & 148 & 81 & 23 & 53 \\ 104 & 95 & 4 & 9 & 285 & 96 & 174 & 37 & 117 \\ 66 & 89 & 2 & 9 & 170 & 68 & 101 & 43 & 127 \\ 81 & 92 & 4 & 9 & 183 & 63 & 103 & 43 & 125 \\ 85 & 97 & 6 & 10 & 183 & 63 & 104 & 42 & 128 \\ 85 & 92 & 2 & 9 & 261 & 63 & 101 & 38 & 110 \\ 68 & 81 & 2 & 8 & 258 & 79 & 98 & 39 & 111 \\ 68 & 87 & 2 & 9 & 257 & 77 & 98 & 38 & 110 \\ 90 & 92 & 3 & 9 & 257 & 78 & 103 & 39 & 112 \\ 62 & 84 & 2 & 8 & 243 & 80 & 101 & 39 & 112 \\ 81 & 96 & 2 & 9 & 257 & 77 & 103 & 39 & 113 \\ 82 & 111 & 3 & 10 & 167 & 66 & 115 & 48 & 153 \\ 79 & 100 & 3 & 9 & 170 & 67 & 105 & 46 & 141 \\ 94 & 101 & 8 & 10 & 163 & 75 & 104 & 45 & 141 \\ & & & & & & & & \end{array}$


Table 4. (Continued).

\begin{tabular}{ccccccccc}
$\begin{array}{c}\text { Sample } \\
\text { (interval in cm) }\end{array}$ & $\mathrm{Cu}$ & $\mathrm{Zn}$ & $\mathrm{Rb}$ & $\begin{array}{c}\mathrm{Nb} \\
\begin{array}{c}\text { (ppm) } \\
\mathrm{Cr}\end{array}\end{array}$ & $\mathrm{Ni}$ & $\mathrm{Sr}$ & $\mathrm{Y}$ & $\mathrm{Zr}$ \\
\hline
\end{tabular}

Hole 483 (Cont.)

$\begin{array}{lrrrrrrrrr}26-1,59-63 & 75 & 103 & 2 & 10 & 166 & 65 & 107 & 46 & 140 \\ 26-2,40-44 & 76 & 100 & 2 & 9 & 164 & 64 & 106 & 46 & 137 \\ 26-3,124-130 & 65 & 96 & 1 & 10 & 153 & 68 & 107 & 48 & 144\end{array}$

Hole 483B

\begin{tabular}{|c|c|c|c|c|c|c|c|c|c|}
\hline $4-1,26-30$ & 88 & 70 & 4 & 8 & 351 & 64 & 111 & 28 & 74 \\
\hline $7-1,7-12$ & 77 & 84 & . & 7 & 226 & 50 & 93 & 32 & 78 \\
\hline$-1,26-31$ & 127 & 92 & 3 & 9 & 233 & 52 & 91 & 31 & 74 \\
\hline $7-2,56-61$ & 80 & 79 & 4 & 9 & 220 & 55 & 93 & 31 & 75 \\
\hline $7-3,2-6$ & 80 & 80 & 1 & 6 & 210 & 53 & 95 & 32 & 75 \\
\hline $8-1,29-35$ & 100 & 71 & 0 & 8 & 320 & 109 & 118 & 25 & 60 \\
\hline $8-1,55-61$ & 82 & 71 & ( & 8 & 294 & 102 & 90 & 23 & 58 \\
\hline $8-1,120-126$ & 87 & 73 & 2 & 8 & 325 & 104 & 93 & 25 & 59 \\
\hline $8-2,121-126$ & 108 & 76 & 2 & 7 & 301 & 87 & 165 & 26 & 63 \\
\hline $8-7,2-20$ & 94 & 66 & & 0 & 290 & 114 & 132 & 26 & 65 \\
\hline $12-1,92-95$ & 71 & 87 & 3 & 7 & 189 & 74 & 115 & 36 & 98 \\
\hline $13-1,38-44$ & 70 & 93 & 6 & 10 & 182 & 67 & 102 & 42 & 125 \\
\hline $13-1,60-64$ & 72 & 95 & 2 & 10 & 182 & 63 & 103 & 43 & 126 \\
\hline $13-1,60-64$ & 83 & 98 & 3 & 9 & 187 & 66 & 105 & 43 & 129 \\
\hline $13-1,66-67$ & 85 & 89 & 8 & 0 & 182 & 84 & 95 & 48 & 142 \\
\hline $13-1,66-67$ & 59 & 93 & 2 & 10 & 185 & 58 & 100 & 43 & 127 \\
\hline $13-1,74-79$ & 65 & 87 & 5 & 9 & 180 & 54 & 101 & 43 & 126 \\
\hline $13-1,92-98$ & 71 & 91 & 2 & 9 & 214 & .73 & 98 & 40 & 116 \\
\hline $13-1,108-119$ & 65 & 94 & 2 & 10 & 182 & 62 & 99 & 42 & 122 \\
\hline $13-1,115-122$ & 65 & 94 & 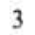 & 8 & 169 & 59 & 102 & 43 & 128 \\
\hline $13-1,115-1$ & 61 & 93 & & . & 189 & 64 & 103 & 42 & 124 \\
\hline $13-1,124-1$ & 55 & 96 & 1 & 10 & 194 & 66 & 103 & 42 & 124 \\
\hline $13-1,124-131$ & 99 & 96 & 7 & 8 & 190 & 65 & 101 & 42 & 125 \\
\hline $13-1,134-1$ & 79 & 95 & & 9 & 186 & 65 & 103 & 43 & 130 \\
\hline $14-1,130-133$ & 88 & 91 & & 9 & 207 & 81 & 102 & 40 & 118 \\
\hline $18-1,49-52$ & 76 & 108 & & 0 & 152 & 56 & 105 & 47 & 148 \\
\hline $18-1,103-107$ & 70 & 94 & 5 & 10 & 152 & 66 & 104 & 45 & 139 \\
\hline $18-2,18-22$ & 81 & 103 & & 10 & 166 & 66 & 106 & 47 & 142 \\
\hline $19-2,17-21$ & 73 & 105 & & 9 & 159 & 59 & 107 & 47 & 142 \\
\hline $19-2,104-108$ & 67 & 103 & & 10 & 158 & 68 & 110 & 48 & 149 \\
\hline $19-3,8-12$ & 69 & 94 & & 10 & 163 & 77 & 113 & 46 & 143 \\
\hline $20-1,14-19$ & 64 & 102 & 1 & 9 & 164 & 89 & 107 & 44 & 139 \\
\hline $20-1,94-99$ & 78 & 102 & 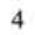 & 10 & 175 & 86 & 110 & 45 & 140 \\
\hline $20-2,9-14$ & 79 & 98 & & 10 & 180 & 75 & 106 & 45 & 138 \\
\hline $20-2$ & 80 & 99 & & 0 & 186 & 70 & 103 & 46 & 136 \\
\hline $20-2$ & 85 & 107 & & 10 & 170 & 69 & 106 & 45 & 138 \\
\hline $20-2,37-42$ & 83 & 102 & & 10 & 162 & 68 & 103 & 46 & 139 \\
\hline $20-2,50-59$ & 67 & 100 & & 10 & 171 & 72 & 109 & 46 & 145 \\
\hline $20-2,50-59$ & 68 & 103 & & 10 & 168 & 71 & 105 & 46 & 141 \\
\hline $20-2,50-59$ & 88 & 114 & 3 & 10 & 170 & 66 & 109 & 48 & 144 \\
\hline $20-2$ & 59 & 100 & & 0 & 227 & 73 & 101 & 44 & 130 \\
\hline $21-2,5$ & 8 & 93 & & 10 & 206 & 70 & 112 & 39 & 110 \\
\hline $22-1,26-32$ & 86 & 98 & & 9 & 189 & 64 & 111 & 39 & 109 \\
\hline $22-2,63-67$ & 86 & 85 & 3 & 7 & 214 & 77 & 112 & 36 & 100 \\
\hline $22-2,7$ & 69 & 90 & 1 & 9 & 196 & 70 & 108. & 37 & 103 \\
\hline $23-4,19-24$ & 84 & 88 & 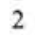 & 9 & 211 & 74 & 109 & 35 & 102 \\
\hline $25-2,22-28$ & 75 & 101 & 1 & 0 & 155 & 60 & 119 & 43 & 122 \\
\hline $25-2,39-45$ & 80 & 96 & 4 & 9 & 177 & 63 & 113 & 40 & 116 \\
\hline $26-2,4$ & 66 & 90 & & 0 & 165 & 61 & 108 & 40 & 105 \\
\hline $26-2,1$ & 73 & 86 & 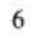 & 10 & 17 & 60 & 109 & 39 & 105 \\
\hline $27-1,12-18$ & 82 & 92 & 4 & 9 & 184 & 66 & 119 & 38 & 116 \\
\hline $27-1,114-11$ & 67 & 93 & & - & 168 & 62 & 110 & 38 & 112 \\
\hline $27-2,95-10$ & 68 & 93 & 4 & 10 & 163 & 60 & 108 & 39 & 115 \\
\hline $27-3,9$ & 63 & 92 & 1 & 10 & 142 & 54 & 105 & 39 & 110 \\
\hline $27-4,7$ & 76 & 91 & & 9 & 213 & 67 & 107 & 38 & 110 \\
\hline $28-1,42-59$ & 56 & 83 & 3 & 9 & 217 & 74 & 106 & 38 & 110 \\
\hline $28-1,123-12$ & 76 & 82 & 3 & 8 & 246 & 84 & 105 & 36 & 105 \\
\hline 28 & 65 & 82 & 2 & 10 & & 91 & 104 & 36 & 101 \\
\hline $28-3,37-40$ & 74 & 82 & 4 & 8 & 251 & 95 & 104 & 36 & 102 \\
\hline $29-1$ & 78 & 85 & 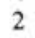 & 9 & 232 & 88 & 105 & 37 & 105 \\
\hline $29-1$ & 69 & 81 & 2 & 0 & 204 & 77 & 108 & 38 & 109 \\
\hline
\end{tabular}

Table 4. (Continued).

\begin{tabular}{llllllllll}
\hline $\begin{array}{c}\text { Sample } \\
\text { (interval in } \mathrm{cm} \text { ) }\end{array}$ & $\mathrm{Cu}$ & $\mathrm{Zn}$ & $\mathrm{Rb}$ & $\begin{array}{c}\mathrm{Nb} \\
\begin{array}{c}\mathrm{(ppm}) \\
\mathrm{Cr}\end{array}\end{array}$ & $\mathrm{Ni}$ & $\mathrm{Sr}$ & $\mathrm{Y}$ & $\mathrm{Zr}$ \\
\hline
\end{tabular}

Hole 483B (Cont.)

$\begin{array}{lrrrrrrrrr}29-1,40-45 & 62 & 90 & 2 & 8 & 201 & 68 & 108 & 38 & 108 \\ 29-1,55-57 & 84 & 94 & 0 & 10 & 187 & 68 & 110 & 38 & 112 \\ 29-1,57-62 & 99 & 98 & 6 & 10 & 210 & 79 & 123 & 40 & 121 \\ 29-1,57-62 & 56 & 88 & 1 & 9 & 192 & 66 & 111 & 40 & 111 \\ 30-3,13-16 & 70 & 89 & 1 & 9 & 230 & 82 & 111 & 39 & 110 \\ 30-3,18-22 & 67 & 84 & 3 & 10 & 232 & 80 & 110 & 39 & 111 \\ 31-3,20-25 & 72 & 85 & 2 & 8 & 298 & 106 & 120 & 38 & 104 \\ 32-1,1-6 & 67 & 80 & 6 & 9 & 296 & 117 & 115 & 34 & 99 \\ 32-1,27-31 & 64 & 77 & 3 & 9 & 293 & 97 & 119 & 35 & 100 \\ 32-1,44-50 & 80 & 88 & 3 & 8 & 300 & 104 & 116 & 35 & 100 \\ 32-1,90-94 & 84 & 100 & 2 & 10 & 298 & 103 & 117 & 36 & 101 \\ 32-1,90-94 & 74 & 84 & 2 & 10 & 303 & 111 & 118 & 36 & 102 \\ 32-1,84-88 & 72 & 82 & 3 & 8 & 311 & 133 & 118 & 34 & 99\end{array}$

Hole $483 \mathrm{C}$

$\begin{array}{llllllllll}4-4,58-66 & 70 & 81 & 5 & 8 & 166 & 60 & 87 & 35 & 96\end{array}$

Hole $485 \mathrm{~A}$

\begin{tabular}{|c|c|c|c|c|c|c|c|c|c|}
\hline $11-3,57-60$ & 122 & 94 & 2 & 8 & 423 & 244 & 181 & 35 & 98 \\
\hline $11-3,68-73$ & 84 & 69 & 4 & 7 & 392 & 208 & 108 & 31 & 91 \\
\hline $11-3,116-121$ & 79 & 71 & 2 & 6 & 417 & 233 & 184 & 32 & 92 \\
\hline $12-1,10-16$ & 55 & 98 & 1 & 8 & 160 & 62 & 95 & 46 & 124 \\
\hline $12-1,76-82$ & 56 & 101 & 0 & 9 & 155 & 58 & 90 & 46 & 126 \\
\hline $12-1,114-120$ & 70 & 99 & 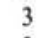 & 8 & 157 & 60 & 92 & 45 & 124 \\
\hline $13-1,11-18$ & 70 & 99 & 2 & 9 & 172 & 71 & 96 & 45 & 119 \\
\hline $13-1,110-117$ & 50 & 95 & 1 & 6 & 157 & 69 & 101 & 45 & 120 \\
\hline $17-1,77-82$ & 44 & 87 & 3 & 10 & 221 & 86 & 146 & 42 & 136 \\
\hline $23-1,50-56$ & 52 & 54 & 8 & 8 & 198 & 68 & 110 & 47 & 141 \\
\hline $23-1,50-56$ & 97 & 78 & 13 & 9 & 199 & 75 & 123 & 45 & 139 \\
\hline $23-1,50-56$ & 64 & 78 & 4 & 9 & 215 & 76 & 112 & 48 & 144 \\
\hline $23-1,148-149$ & 47 & 94 & 2 & 8 & 212 & 70 & 107 & 46 & 136 \\
\hline$-2,16-22$ & 79 & 94 & 4 & 10 & 220 & 72 & 111 & 47 & 137 \\
\hline$-3,120-125$ & 59 & 96 & 2 & 9 & 250 & 71 & 110 & 44 & 137 \\
\hline $25-1,117-143$ & 58 & 94 & 2 & 10 & 217 & 77 & 102 & 46 & 135 \\
\hline $29-1,2-8$ & 80 & 111 & 5 & 8 & 216 & 99 & 117 & 46 & 141 \\
\hline$-1,89-93$ & 60 & 81 & 4 & 10 & 226 & 90 & 108 & 45 & 142 \\
\hline $29-2,143-148$ & 91 & 98 & 5 & 10 & 191 & 67 & 103 & 43 & 130 \\
\hline $29-3,10-16$ & 63 & 80 & 7 & 8 & 266 & 77 & 95 & 44 & 139 \\
\hline $30-2,96-101$ & 107 & 110 & 5 & 10 & 329 & 57 & 91 & 48 & 146 \\
\hline $0-3,38-41$ & 78 & 78 & 4 & 9 & 651 & 86 & 90 & 39 & 112 \\
\hline$-3,44-49$ & 31 & 66 & 2 & 7 & 526 & 79 & 97 & 40 & 108 \\
\hline$-4,8-13$ & 28 & 77 & 3 & 9 & 160 & 78 & 107 & 41 & 127 \\
\hline $1-1,62-67$ & 58 & 86 & 1 & 9 & 405 & 96 & 89 & 34 & 90 \\
\hline $1-3,34-39$ & 63 & 109 & 4 & 7 & 222 & 101 & 100 & 41 & 117 \\
\hline $2-1,55-61$ & 45 & 55 & 5 & 10 & 180 & 66 & 110 & 48 & 141 \\
\hline $32-2,99-105$ & 64 & 105 & 5 & 9 & 180 & 129 & 99 & 42 & 123 \\
\hline $32-3,47-51$ & 73 & 104 & 4 & 7 & 252 & 124 & 98 & 41 & 120 \\
\hline $32-5,9-14$ & 44 & 90 & 4 & 9 & 240 & 123 & 98 & 43 & 128 \\
\hline $32-6,52-57$ & 73 & 103 & 5 & 10 & 198 & 110 & 97 & 46 & 137 \\
\hline $3-1,2-9$ & 68 & 121 & 6 & 8 & 216 & 111 & 94 & 46 & 137 \\
\hline $33-1,74-79$ & 52 & 123 & 5 & 10 & 223 & 120 & 96 & 45 & 134 \\
\hline $33-2,26-33$ & 72 & 125 & 10 & 8 & 234 & 121 & 97 & 45 & 127 \\
\hline $33-2,72-78$ & 74 & 97 & 9 & 8 & 220 & 104 & 92 & 44 & 137 \\
\hline $33-2,88-94$ & 65 & 96 & 5 & 10 & 228 & 100 & 101 & 47 & 140 \\
\hline $34-1,86-90$ & 74 & 90 & 1 & 9 & 269 & 71 & 102 & 88 & 113 \\
\hline $34-2,96-102$ & 65 & 85 & 1 & 7 & 256 & 69 & 95 & 39 & 110 \\
\hline $35-1,135-142$ & 72 & 86 & 3 & 8 & 250 & 71 & 95 & 38 & 106 \\
\hline $35-2,113-118$ & 70 & 83 & 3 & 8 & 248 & 71 & 95 & 9 & 109 \\
\hline $35-3,21-27$ & 60 & 74 & 2 & 7 & 240 & 69 & 133 & 38 & 109 \\
\hline $35-4,85-92$ & 70 & 88 & 2 & 10 & 260 & 70 & 92 & 39 & 109 \\
\hline $35-6,47-53$ & 70 & 87 & 2 & 8 & 254 & 67 & 95 & 40 & 111 \\
\hline $36-3,65-69$ & 91 & 161 & 2 & 7 & 251 & 107 & 144 & 44 & 140 \\
\hline $38-3,18-23$ & 52 & 89 & 3 & 8 & 212 & 84 & 102 & 39 & 109 \\
\hline $38-6,58-63$ & 61 & 87 & 4 & 9 & 200 & 81 & 101 & 41 & 118 \\
\hline $39-1,26-32$ & 40 & 81 & 2 & 9 & 222 & 76 & 104 & 40 & 114 \\
\hline $39-1,59-64$ & 70 & 94 & 7 & 8 & 202 & 77 & 95 & 39 & 114 \\
\hline
\end{tabular}


Table 5. Trace element ratios in fresh basalts from DSDP Sites 482,483 , and 485

\begin{tabular}{|c|c|}
\hline $\begin{array}{l}\text { Sample } \\
\text { (interval in } \mathrm{cm} \text { ) }\end{array}$ & $1 / \mathrm{Ni}$ \\
\hline Hole $482 \mathrm{~B}$ & \\
\hline $13-1,99-104$ & 14.29 \\
\hline $14-1,91-94$ & 9.80 \\
\hline $14-1,132-137$ & 11.11 \\
\hline $14-3,2-6$ & 11.11 \\
\hline $14-4,16-20$ & 12.00 \\
\hline $15-1,12-16$ & 10.99 \\
\hline $16-4,100-104$ & 17.54 \\
\hline $20-1,27-30$ & 13.15 \\
\hline $20-3,144-147$ & 13.04 \\
\hline $21-2,19-23$ & 17.86 \\
\hline $21-3,58-63$ & 17.24 \\
\hline $22-2,67-72$ & 16.13 \\
\hline $22-2,130-136$ & 17.54 \\
\hline $22-3,103-107$ & 14.08 \\
\hline $22-4,97-102$ & 14.92 \\
\hline $24-3,110-115$ & 15.87 \\
\hline Hole $482 \mathrm{C}$ & \\
\hline $10-1,0-4$ & 16.39 \\
\hline $10-1.9-12$ & 14.93 \\
\hline $10-1,12-16$ & 15.63 \\
\hline $10-3,11-14$ & 13.70 \\
\hline $11-3,65-69$ & 14.08 \\
\hline $12-1,92-118$ & 17.54 \\
\hline $13-1,10-15$ & 17.24 \\
\hline $14-1,13-18$ & 18.52 \\
\hline $15-1,114-118$ & 18.18 \\
\hline Hole 482D & \\
\hline $8-1,24-29$ & 14.08 \\
\hline $8-1,65-72$ & 14.49 \\
\hline $10-1,106-109$ & 10.99 \\
\hline $10-1,111-114$ & 13.70 \\
\hline $10-2,70-72$ & 15.15 \\
\hline $10-2,128-130$ & 15.15 \\
\hline $10-3,25-27$ & 15.87 \\
\hline $10-3,93-97$ & 10.75 \\
\hline $12-1,28-32$ & 11,11 \\
\hline $12-3,27-31$ & 18.18 \\
\hline $13-1,49-51$ & 17.86 \\
\hline $13-1,98-101$ & 17.54 \\
\hline $13-1,128-134$ & 16.39 \\
\hline Hole $482 \mathrm{~F}$ & \\
\hline $5-1,133-139$ & 15.63 \\
\hline $11-1,125-128$ & 10.75 \\
\hline Hole 483 & \\
\hline $14-1,58-68$ & 16.13 \\
\hline $15-1,93-97$ & 15.38 \\
\hline $16-2,64-70$ & 19.23 \\
\hline $16-2,99-100$ & 18.52 \\
\hline $16-3,2-6$ & 17.86 \\
\hline $17-2,13-17$ & 9.26 \\
\hline $17-2,17-21$ & 6.76 \\
\hline $18-4,131-134$ & 10.42 \\
\hline $21-1,49-55$ & 14.71 \\
\hline $21-1,57-61$ & 15.87 \\
\hline $21 \cdot 3,6-9$ & 15.87 \\
\hline $22-2,68-72$ & 15.87 \\
\hline $22-2,74-77$ & 12.66 \\
\hline $22-2,85-88$ & 12.99 \\
\hline $22-2,88-93$ & 12.82 \\
\hline $22-2,94-99$ & 12.50 \\
\hline $23-1,10-16$ & 12.99 \\
\hline $24-1,52-57$ & 15.15 \\
\hline $24-2,35-40$ & 14.93 \\
\hline $25 \cdot 2,61-66$ & 13.33 \\
\hline $26-1,59-63$ & 15.38 \\
\hline $26-2,40-44$ & 15.63 \\
\hline $26-3,124-130$ & 17.24 \\
\hline Hole 483B & \\
\hline $4-1.26-30$ & 15.63 \\
\hline $7-1,7-12$ & 20.00 \\
\hline $7-1,26-31$ & 19.23 \\
\hline $7 \cdot 2,56-61$ & 18.18 \\
\hline $7-3,2-6$ & 18.87 \\
\hline $8-1,29-35$ & 9.17 \\
\hline $8-1,55-61$ & 9.80 \\
\hline $8-1,120-126$ & 9.62 \\
\hline $8-2,121-126$ & 9.49 \\
\hline 8-7, $2-20$ & 8.77 \\
\hline $12-1,92-95$ & 13.51 \\
\hline $13-1,38-44$ & 14.93 \\
\hline $13-1,60-64$ & 15.88 \\
\hline $13-1,60-64$ & 15.15 \\
\hline $13-1,66-67$ & 11.90 \\
\hline $13-1,36-67$ & 17.24 \\
\hline $13 \cdot 1,74-79$ & 18.52 \\
\hline $13-1,92-98$ & 13.70 \\
\hline $13 \cdot 1,108-119$ & 16.13 \\
\hline $13-1.115-122$ & 16.95 \\
\hline $13-1,115-122$ & 15.63 \\
\hline $13-1,124-131$ & 15.15 \\
\hline $13-1,124-131$ & 15.38 \\
\hline $13-1,134-13$ & 15 \\
\hline $14-1,134-139$ & 12.35 \\
\hline $18-1,49-52$ & 18.18 \\
\hline $18-1,103-107$ & 15.15 \\
\hline
\end{tabular}

Table 5. (Continued).

\begin{tabular}{|c|c|c|c|c|c|c|c|c|c|}
\hline $\begin{array}{c}\text { Sample } \\
\text { (interval in } \mathrm{cm} \text { ) }\end{array}$ & $1 / \mathrm{Ni}$ & $1 / \mathrm{St}$ & $1 / Y$ & $\mathrm{Zr} / \mathrm{Y}$ & $\mathrm{Zr} / \mathrm{Nb}$ & $\mathrm{Ti} / \mathrm{Y}$ & $\mathrm{Zr} / \mathrm{SR}$ & $\mathrm{Zr} / \mathrm{Ni}$ & $\mathrm{Y} / \mathrm{Nb}$ \\
\hline \multicolumn{10}{|c|}{ Hole $483 \mathrm{~B}$ (Cont.) } \\
\hline $18-2,18-22$ & 15.15 & 9.43 & 21.28 & 3.02 & 14.20 & 270.6 & 34 & 2.15 & 70 \\
\hline 2. 17-21 & 16.95 & $\begin{array}{r}9.35 \\
0.09\end{array}$ & $\begin{array}{l}21.28 \\
2083\end{array}$ & 3.02 & 15.78 & 268.1 & 1.33 & 2.41 & 5.22 \\
\hline $\begin{array}{l}19-2.2 .104-108 \\
19-3,8-12\end{array}$ & $\begin{array}{l}14.71 \\
12.99\end{array}$ & $\begin{array}{l}9.09 \\
8.85\end{array}$ & $\begin{array}{l}\begin{array}{l}20.83 \\
21.74\end{array} \\
2.0\end{array}$ & $\begin{array}{l}3.10 \\
3.11\end{array}$ & $\begin{array}{l}14.90 \\
14.30\end{array}$ & $\begin{array}{l}\begin{array}{l}273.6 \\
274.0\end{array} \\
\end{array}$ & $\begin{array}{l}1.35 \\
1.27\end{array}$ & $\begin{array}{l}2.19 \\
1.86\end{array}$ & $\begin{array}{l}4.80 \\
4.60\end{array}$ \\
\hline $\begin{array}{l}19-3,8-12 \\
20-1,14-19\end{array}$ & $\begin{array}{l}12.99 \\
11.24\end{array}$ & $\begin{array}{l}8.85 \\
9.35\end{array}$ & $\begin{array}{l}21.74 \\
22.73\end{array}$ & $\begin{array}{l}3.11 \\
3.16\end{array}$ & $\begin{array}{l}15,30 \\
15,44\end{array}$ & 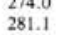 & 1.30 & $\begin{array}{l}1.86 \\
1.56\end{array}$ & $\begin{array}{l}4.60 \\
4.89\end{array}$ \\
\hline $20-1,94-99$ & 11.63 & 9.09 & 22.22 & 3.11 & 14.00 & 273.5 & 1.27 & 1.63 & 4.50 \\
\hline $20-2,9-14$ & 13.33 & 9.43 & 22.22 & 3.07 & 13.80 & 270.9 & 1.30 & 1.84 & 4.50 \\
\hline 2. $16-21$ & 14.29 & 9.71 & 21.74 & 2.96 & 17,00 & 262.4 & 1.28 & 1.94 & 5.75 \\
\hline $\begin{array}{l}-2,25-30 \\
-2,37-42\end{array}$ & $\begin{array}{l}14.49 \\
14.71\end{array}$ & $\begin{array}{l}9.43 \\
9.77\end{array}$ & $\begin{array}{l}22.22 \\
21.74\end{array}$ & $\begin{array}{l}3.07 \\
3.02\end{array}$ & $\begin{array}{l}13.80 \\
13.90\end{array}$ & $\begin{array}{l}277.4 \\
270.4\end{array}$ & $\begin{array}{l}1.31 \\
1.35\end{array}$ & $\begin{array}{l}2.00 \\
2.04\end{array}$ & $\begin{array}{l}4.50 \\
4.60\end{array}$ \\
\hline $\begin{array}{l}20-2,37-42 \\
20-2,50-59\end{array}$ & $\begin{array}{l}\begin{array}{l}14.71 \\
13.89\end{array} \\
13\end{array}$ & $\begin{array}{l}9.71 \\
9.17\end{array}$ & $\begin{array}{l}\begin{array}{l}21.74 \\
21.74\end{array} \\
2\end{array}$ & $\begin{array}{l}3.02 \\
3.15\end{array}$ & $\begin{array}{l}13.90 \\
14.50\end{array}$ & $\begin{array}{l}\begin{array}{l}270.1 \\
280.4\end{array} \\
2\end{array}$ & $\begin{array}{l}1.35 \\
1.33\end{array}$ & $\begin{array}{l}2.04 \\
2.01\end{array}$ & $\begin{array}{l}4.60 \\
4.60\end{array}$ \\
\hline $20-2,50-59$ & 14.08 & 9.52 & 21.74 & 3.07 & 14.10 & 274.0 & 1.34 & 1.99 & 4.60 \\
\hline $20-2,50-59$ & 15.15 & 9.17 & 20.83 & 3.00 & 14.40 & 263.8 & 1.32 & 2.18 & 4.80 \\
\hline$=2,142-146$ & $\begin{array}{l}13.70 \\
14.9\end{array}$ & 9.90 & 22.73 & 2.95 & 14.44 & 270.4 & $\begin{array}{l}1.29 \\
0.98\end{array}$ & 1.78 & 4.89 \\
\hline $\begin{array}{l}2,57-56 \\
1.26-32\end{array}$ & $\begin{array}{l}14.29 \\
1563\end{array}$ & $\begin{array}{l}8.93 \\
900\end{array}$ & $\begin{array}{l}25.64 \\
25.64\end{array}$ & $\begin{array}{l}2.82 \\
2.79\end{array}$ & 11.00 & $\begin{array}{l}268.8 \\
2703\end{array}$ & $\begin{array}{l}0.98 \\
0.98\end{array}$ & $\begin{array}{l}1.57 \\
1.70\end{array}$ & $\begin{array}{l}3,90 \\
4.33\end{array}$ \\
\hline $\begin{array}{l}-1,26-32 \\
-2,63-67\end{array}$ & $\begin{array}{l}15.63 \\
12.99\end{array}$ & $\begin{array}{l}9.01 \\
8.93\end{array}$ & $\begin{array}{l}25.64 \\
27.78\end{array}$ & $\begin{array}{l}2.79 \\
2.78\end{array}$ & $\begin{array}{l}12.11 \\
14.29\end{array}$ & $\begin{array}{l}\begin{array}{l}270.3 \\
263.4\end{array} \\
\end{array}$ & $\begin{array}{l}0.98 \\
0.89\end{array}$ & $\begin{array}{l}1.70 \\
1.30\end{array}$ & $\begin{array}{l}4.33 \\
5.14\end{array}$ \\
\hline $22-2,77-82$ & 14.29 & 9.71 & 27.03 & 2.78 & 11.44 & 264.2 & 1.00 & 1.47 & 4.11 \\
\hline $23-4,19-24$ & 13.51 & 9.17 & 28.57 & 2.91 & 11.22 & 281.0 & 0.94 & 1.38 & 3.89 \\
\hline $\begin{array}{l}25-2,22-28 \\
25,239.45\end{array}$ & $\begin{array}{l}16.67 \\
11587\end{array}$ & $\begin{array}{l}8.40 \\
8.85\end{array}$ & $\begin{array}{l}23.26 \\
2500\end{array}$ & 2.84 & $\begin{array}{l}13.56 \\
2.89\end{array}$ & $\begin{array}{l}269.8 \\
270.9\end{array}$ & 1.03 & 2.03 & 4.78 \\
\hline $\begin{array}{l}25-2,39-45 \\
26-2,42-47\end{array}$ & $\begin{array}{l}15.87 \\
16.39\end{array}$ & $\begin{array}{l}8.85 \\
9.26\end{array}$ & $\begin{array}{l}25.00 \\
25.00\end{array}$ & $\begin{array}{l}2.90 \\
2.63\end{array}$ & 12.89 & $\begin{array}{l}270.9 \\
2<4.7\end{array}$ & $\begin{array}{l}1.03 \\
0.97\end{array}$ & 1.84 & $\begin{array}{l}4.44 \\
500\end{array}$ \\
\hline $26-2,126-137$ & $\begin{array}{l}16.67 \\
16.67\end{array}$ & 9.17 & 25.64 & $\begin{array}{l}2.63 \\
2.69\end{array}$ & $\begin{array}{l}11.13 \\
10.50\end{array}$ & $\begin{array}{l}254.7 \\
256.7\end{array}$ & 0.96 & 1.75 & $\begin{array}{l}3.90 \\
3.90\end{array}$ \\
\hline $27-1,12-18$ & 15.15 & 8.40 & 26.32 & 3.05 & 12.89 & 288.3 & 0.97 & 1.75 & 4.22 \\
\hline$-1,114-119$ & 16.13 & 9.09 & 26.32 & 2.95 & 12.44 & 283.6 & 1.02 & 1.81 & 4.22 \\
\hline $\begin{array}{l}-2,95-101 \\
3,94-98\end{array}$ & 16.67 & $\begin{array}{l}9.26 \\
0.92\end{array}$ & 15.64 & 2.95 & 11.50 & 280.9 & 1.06 & 1.92 & 3.90 \\
\hline $\begin{array}{l}27-3,94-98 \\
27-4,7480\end{array}$ & $\begin{array}{l}18.52 \\
14.93\end{array}$ & $\begin{array}{l}9.52 \\
9.35\end{array}$ & $\begin{array}{l}25.64 \\
26.32\end{array}$ & $\begin{array}{l}2.82 \\
2.89\end{array}$ & 11.00 & $\begin{array}{l}275.0 \\
271.2\end{array}$ & $\begin{array}{l}1.05 \\
1.03\end{array}$ & 2.04 & $\begin{array}{l}3.90 \\
4.22\end{array}$ \\
\hline $\begin{array}{l}27-4,74-80 \\
28-1,42-59\end{array}$ & $\begin{array}{l}\begin{array}{l}4.93 \\
13.51\end{array} \\
13\end{array}$ & $\begin{array}{l}9.35 \\
9.43\end{array}$ & $\begin{array}{l}26.32 \\
26.32\end{array}$ & $\begin{array}{l}2.89 \\
2.89\end{array}$ & $\begin{array}{l}12.22 \\
12.22\end{array}$ & $\begin{array}{l}271.2 \\
272.8\end{array}$ & $\begin{array}{l}1.03 \\
1.04\end{array}$ & $\begin{array}{l}1.64 \\
1.49\end{array}$ & $\begin{array}{l}4.22 \\
4.22\end{array}$ \\
\hline $28-1,123-128$ & 11.90 & 9.52 & 27.78 & 2.92 & 13.13 & 274.8 & 1.00 & 1.24 & 4.50 \\
\hline $28-2,28-32$ & 10.99 & 9.62 & $\begin{array}{l}27,78 \\
27.78\end{array}$ & 2.81 & 10.10 & 268.3 & 1.00 & 1.11 & 3.60 \\
\hline $28-3.37-40$ & 10.53 & 9.62 & 27.78 & 2.83 & 12.75 & 268.3 & 0.97 & 1.07 & 4.50 \\
\hline $\begin{array}{l}29-1,10-15 \\
29-1.26-31\end{array}$ & $\begin{array}{l}11.36 \\
11.99\end{array}$ & $\begin{array}{l}9.52 \\
9.96\end{array}$ & $\begin{array}{l}27.03 \\
26.32\end{array}$ & $\begin{array}{l}2.84 \\
2.87\end{array}$ & 11.67 & $\begin{array}{l}267.4 \\
269.7\end{array}$ & $\begin{array}{l}0.98 \\
1.00\end{array}$ & $\begin{array}{l}1.19 \\
1.42\end{array}$ & 4.11 \\
\hline $\begin{array}{l}29-1,26-231 \\
29-1,40-45\end{array}$ & $\begin{array}{l}12.99 \\
14.71\end{array}$ & $\begin{array}{l}9.26 \\
9.26\end{array}$ & $\begin{array}{l}26.32 \\
26.32\end{array}$ & $\begin{array}{l}2.87 \\
2.84\end{array}$ & $\begin{array}{l}12.11 \\
13.50\end{array}$ & $\begin{array}{l}269.7 \\
271.2\end{array}$ & $\begin{array}{l}1.00 \\
1.01\end{array}$ & $\begin{array}{l}1.42 \\
1.59\end{array}$ & $\begin{array}{l}4.22 \\
4.75\end{array}$ \\
\hline $29-1,55-57$ & 14.71 & 9.09 & 26.32 & 2.95 & 11.20 & 277.4 & 1.00 & 1.65 & 3.80 \\
\hline $29-1,57-62$ & 12.66 & 8.13 & 25,00 & 3.02 & 12.10 & & 1.02 & .53 & 4.00 \\
\hline $4,57-62$ & 15.15 & 9.01 & $\begin{array}{l}25.00 \\
25.64\end{array}$ & 2.77 & 12.33 & $\begin{aligned} 263.5 \\
2658\end{aligned}$ & 0.98 & 1.62 & 4.44 \\
\hline $\begin{array}{l}30-3.13-16 \\
030-3,18-22\end{array}$ & 12.20 & 9.01 & 25.64 & $\begin{array}{l}2.82 \\
2.85\end{array}$ & 12.22 & $\begin{array}{l}265.8 \\
267.3\end{array}$ & 0.99 & $\begin{array}{l}1.34 \\
39\end{array}$ & $\begin{array}{r}4.33 \\
3.90\end{array}$ \\
\hline $\begin{array}{l}18-22 \\
20-25\end{array}$ & $\begin{array}{r}12.50 \\
9.43\end{array}$ & $\begin{array}{l}9.09 \\
8.33\end{array}$ & $\begin{array}{l}25.64 \\
26.32\end{array}$ & $\begin{array}{l}2.85 \\
2.74\end{array}$ & $\begin{array}{l}11.10 \\
13,00\end{array}$ & $\begin{array}{l}{ }_{267.3}^{258.8} \\
258 .\end{array}$ & $\begin{array}{l}1.01 \\
0.87\end{array}$ & 98 & $\begin{array}{l}3.90 \\
4.75\end{array}$ \\
\hline $32-1,1-6$ & 8.55 & 8.70 & 29.41 & 2.91 & 11.00 & 273.7 & 0.87 & 0.85 & 3.78 \\
\hline $32-1,27-31$ & 10.31 & 8.40 & 28.57 & 2.86 & & 262.5 & 0.84 & 3 & 3.89 \\
\hline $32-1,44-50$ & 9.62 & $\begin{array}{l}8.62 \\
8 \leqslant 5\end{array}$ & 28.57 & 2.81 & & 262.5 & 0.85 & 1.02 & 4.38 \\
\hline $\begin{array}{l}32-1,90-94 \\
32-1,90-94\end{array}$ & $\begin{array}{l}9.71 \\
9.01\end{array}$ & $\begin{array}{l}8.55 \\
8.47\end{array}$ & $\begin{array}{l}27.78 \\
27.78\end{array}$ & $\begin{array}{l}2.81 \\
2.83\end{array}$ & $\begin{array}{l}10.10 \\
10.20\end{array}$ & $\begin{array}{l}261.7 \\
260.1\end{array}$ & $\begin{array}{l}0.86 \\
0.86\end{array}$ & $\begin{array}{l}0.98 \\
0.92\end{array}$ & $\begin{array}{l}3.60 \\
3.60\end{array}$ \\
\hline $32-1,84-88$ & 7.52 & 8.47 & 29.41 & 2.91 & 12.38 & 271.9 & 0.84 & 0.74 & 4.25 \\
\hline \multicolumn{10}{|l|}{ Hole $483 \mathrm{C}$} \\
\hline $4-4,58-66$ & 16.67 & 11.49 & 28.57 & 2.74 & 12.00 & 222.2 & 1.10 & 1.60 & 4.38 \\
\hline Hole $485 \mathrm{~A}$ & & & & & & & & & \\
\hline $11-3,57-60$ & 4.10 & 5.52 & 28.57 & 2.80 & 12.25 & 260.8 & 0.54 & 0.40 & 4.38 \\
\hline$M, \omega_{c}$ & 4.81 & 9.25 & 32.26 & 2.94 & 13.00 & 269.7 & 0.84 & 0.44 & 4.43 \\
\hline $11-3,116-121$ & 4.29 & 5.43 & 31.25 & 2.88 & 15.33 & 268.7 & 0.50 & 0.39 & 5.33 \\
\hline & 16.13 & 10.53 & 21.74 & & & 261.2 & 1.31 & & 75 \\
\hline $\begin{array}{l}12-1,76-82 \\
12-1\end{array}$ & 17.24 & 11.11 & $\begin{array}{l}21.74 \\
2202\end{array}$ & 2.74 & $\begin{array}{l}14.00 \\
1580\end{array}$ & $\begin{array}{l}262.4 \\
265.7\end{array}$ & $\begin{array}{l}1.40 \\
135\end{array}$ & 2.17 & 5.11 \\
\hline $\begin{array}{l}12-1,114-120 \\
13-1.11-18\end{array}$ & $\begin{array}{l}16.66 \\
14.08\end{array}$ & $\begin{array}{l}10.87 \\
10.42\end{array}$ & $\begin{array}{l}22.222 \\
22.22\end{array}$ & $\begin{array}{l}2.76 \\
2.64\end{array}$ & $\begin{array}{l}15.50 \\
13.22\end{array}$ & $\begin{array}{l}265.7 \\
252.6\end{array}$ & $\begin{array}{l}1.35 \\
1.24\end{array}$ & $\begin{array}{l}2.07 \\
1.68\end{array}$ & $\begin{array}{l}5.63 \\
5.00\end{array}$ \\
\hline $\begin{array}{l}\text { 13-1.1. } 1110-117 \\
13-110\end{array}$ & $\begin{array}{l}14,08 \\
14.49\end{array}$ & $\begin{array}{r}0.42 \\
9.90\end{array}$ & 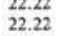 & $\begin{array}{l}2.64 \\
2.67\end{array}$ & $\begin{array}{l}13.22 \\
14.60\end{array}$ & $\begin{array}{l}252.6 \\
257.8\end{array}$ & $\begin{array}{l}1.24 \\
1.19\end{array}$ & $\begin{array}{l}1.68 \\
1.74\end{array}$ & 5.50 \\
\hline $17-1,77-82$ & 11.63 & 6.85 & 23.81 & 3.24 & 13.60 & 300.0 & & & 4. 20 \\
\hline $23.1,50,>0$ & 14.71 & 9.09 & 21.28 & & & 271.9 & & & 5.88 \\
\hline $50-56$ & $\begin{array}{r}13.33 \\
13.16\end{array}$ & $\begin{array}{l}8.13 \\
8.93\end{array}$ & $\begin{array}{l}22.22 \\
20.83\end{array}$ & 3.09 & & $\begin{array}{l}280.0 \\
273.6\end{array}$ & $\begin{array}{l}1.13 \\
1.29\end{array}$ & & $\begin{array}{l}00 \\
33\end{array}$ \\
\hline $\begin{array}{l}23-1,50-56 \\
23-1,148-149\end{array}$ & $\begin{array}{l}13.16 \\
1439\end{array}$ & 8.93 & $\begin{array}{l}20.83 \\
21.74\end{array}$ & 3.00 & $\begin{array}{l}16.00 \\
17.00\end{array}$ & $\begin{array}{l}273.6 \\
271.4\end{array}$ & 1.29 & $\begin{array}{l}1.89 \\
1.94\end{array}$ & $\begin{array}{l}5.33 \\
5.75\end{array}$ \\
\hline $\begin{array}{l}23-1,148-149 \\
23-2,16-22\end{array}$ & $\begin{array}{l}14.89 \\
13.89\end{array}$ & $\begin{array}{l}9.01 \\
9.01\end{array}$ & $\begin{array}{l}11.1 .28 \\
21.28\end{array}$ & 2.91 & 13,70 & 272.7 & 1.23 & 1.90 & 4.70 \\
\hline $23-3,120-125$ & 14.08 & 9.09 & 22.73 & 3.11 & 15.22 & 294.5 & 1.2. & & 4.89 \\
\hline $25-1,117-143$ & 12.99 & 9.80 & 21.74 & 2. & & 270.1 & 1.3 & & 4.60 \\
\hline $2-8$ & 10.10 & 8.55 & $\begin{array}{l}21.74 \\
22.22\end{array}$ & 3.07 & & & 1.21 & & $\begin{array}{l}5.75 \\
4.50\end{array}$ \\
\hline 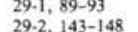 & $\begin{array}{l}11.11 \\
14.93\end{array}$ & $\begin{array}{l}9.26 \\
9.71\end{array}$ & $\begin{array}{l}22.222 \\
23.26\end{array}$ & $\begin{array}{l}3.16 \\
3.02\end{array}$ & $\begin{array}{l}14.20 \\
13.00\end{array}$ & $\begin{array}{l}291.8 \\
265.7\end{array}$ & $\begin{array}{l}1.31 \\
1.26\end{array}$ & $\begin{array}{l}1.58 \\
1.94\end{array}$ & $\begin{array}{l}4.50 \\
4.30\end{array}$ \\
\hline $29 \cdot 3,10-16$ & $\begin{array}{l}4.939 \\
12.99\end{array}$ & 10.53 & 22.73 & $\begin{array}{l}3.02 \\
3.16\end{array}$ & 17.38 & 295.8 & 1.46 & 1.81 & 5.50 \\
\hline $30-2,96-101$ & 17.54 & 10.99 & 20.83 & 3.04 & 14 & 238.0 & 1. & & 4.80 \\
\hline $38-41$ & 11.63 & 11.11 & 25.64 & 2.87 & & 265.8 & & & 4.33 \\
\hline 44 & $\begin{array}{l}12.66 \\
-11.82\end{array}$ & $\begin{array}{l}10.31 \\
9.35\end{array}$ & $\begin{array}{l}25.00 \\
24.39\end{array}$ & 2.70 & 15.43 & $\begin{array}{l}262.1 \\
274.3\end{array}$ & 1.11 & $\begin{array}{l}1.26 \\
1.68\end{array}$ & 5.71 \\
\hline $62-1<-2>$ & $\begin{array}{l}12.82 \\
10.42\end{array}$ & $\begin{array}{r}9.39 \\
11.24\end{array}$ & $\begin{array}{l}\begin{array}{l}24.39 \\
29.41\end{array} \\
\end{array}$ & $\begin{array}{l}3.10 \\
2.65\end{array}$ & $\begin{array}{l}14.11 \\
10.00\end{array}$ & $\begin{array}{l}274,3 \\
251.1\end{array}$ & $\begin{array}{l}1.19 \\
1.01\end{array}$ & $\begin{array}{l}1.68 \\
0.94\end{array}$ & $\begin{array}{l}4.56 \\
3.78\end{array}$ \\
\hline $34-$ & 9.90 & 10.00 & 24.39 & 2.85 & & 268.6 & I. 1 & 1.16 & 5.86 \\
\hline 32. & 15.15 & 9.09 & 20.83 & 2 & & & & & 4.80 \\
\hline $32-2,99-1$ & 7.75 & 10.10 & 23.81 & 2. & & & & & \\
\hline $47-$ & 8.06 & 10.20 & 24.39 & 2.93 & & & & & 86 \\
\hline & $\begin{array}{l}8.13 \\
909\end{array}$ & 10.20 & $\begin{array}{l}23.26 \\
21.74\end{array}$ & $\begin{array}{l}2.98 \\
2.98\end{array}$ & $\begin{array}{l}14.22 \\
13.70\end{array}$ & $\begin{array}{l}273.9 \\
277.8\end{array}$ & $\begin{array}{l}1.31 \\
1.41\end{array}$ & & $\begin{array}{l}4.78 \\
460\end{array}$ \\
\hline & 9.01 & 10.64 & $\begin{array}{l}11.74 \\
21.74\end{array}$ & $\begin{array}{l}2.98 \\
2.98\end{array}$ & & $\begin{array}{l}277.8 \\
271.4\end{array}$ & 1. 2 . & 1.23 & $\begin{array}{l}4.00 \\
5.75\end{array}$ \\
\hline 33. & 8.33 & 10.42 & 22.22 & 2. & & & & & \\
\hline $26-33$ & 8.26 & 10.31 & 22.22 & 2.82 & 15 & 273.5 & 1. & & 3 \\
\hline & 9.62 & 10.87 & 22.73 & 3.11 & 17,1 & 285.1 & 19 & & \\
\hline & 14. & 9. $\Rightarrow$. & 21 & & & 26 & & & $\begin{array}{l}4.70 \\
4.72\end{array}$ \\
\hline 96- & 14. & $\begin{array}{l}9.80 \\
10.53\end{array}$ & & 2.82 & & $\begin{array}{l}282.1 .2 \\
261.2\end{array}$ & & 1.59 & $\begin{array}{l}4.22 \\
5.57\end{array}$ \\
\hline 35 & 14. & 10.53 & 26.32 & 2. & 13 & & I. & & \\
\hline .113 & 14.08 & 10. & 25 & & & 26 & & & 4.88 \\
\hline $35,3+21-2$ & 14.49 & $\begin{array}{l}7.52 \\
0.97\end{array}$ & 26.32 & 2.8 & & 266.6 & & & 13 \\
\hline & 14.29 & 10.87 & $\begin{array}{l}25.64 \\
2500\end{array}$ & 2.79 & 10 & $\begin{array}{l}259.7 \\
2650\end{array}$ & 8 & $\begin{array}{l}1.56 \\
1.66\end{array}$ & $x$ \\
\hline & 9. & & & 3. & & & & 1.31 & 6.29 \\
\hline 18. & 11 & 9. $>>2$ & 25 & 2. & & 264.3 & 1.4 & & 4.88 \\
\hline & 12. & 9. & ${ }_{25}^{24}$ & 2. & & $\begin{array}{l}271.5 \\
272.4\end{array}$ & i. & $\begin{array}{l}1.46 \\
1.50\end{array}$ & $\begin{array}{l}4.50 \\
4.44\end{array}$ \\
\hline $\begin{array}{r}39-1,1 \\
39-69\end{array}$ & $\begin{array}{l}13.16 \\
12.99\end{array}$ & $\begin{array}{r}9.62 \\
10.53\end{array}$ & 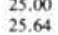 & $\begin{array}{l}2.85 \\
2.92\end{array}$ & $\begin{array}{l}12.67 \\
14.25\end{array}$ & $\begin{array}{l}\begin{array}{l}272.4 \\
280.9\end{array} \\
\text { ? }\end{array}$ & $\begin{array}{l}1.10 \\
1.20\end{array}$ & $\begin{array}{l}1.50 \\
1.48\end{array}$ & $\begin{array}{l}4.44 \\
4.88\end{array}$ \\
\hline
\end{tabular}



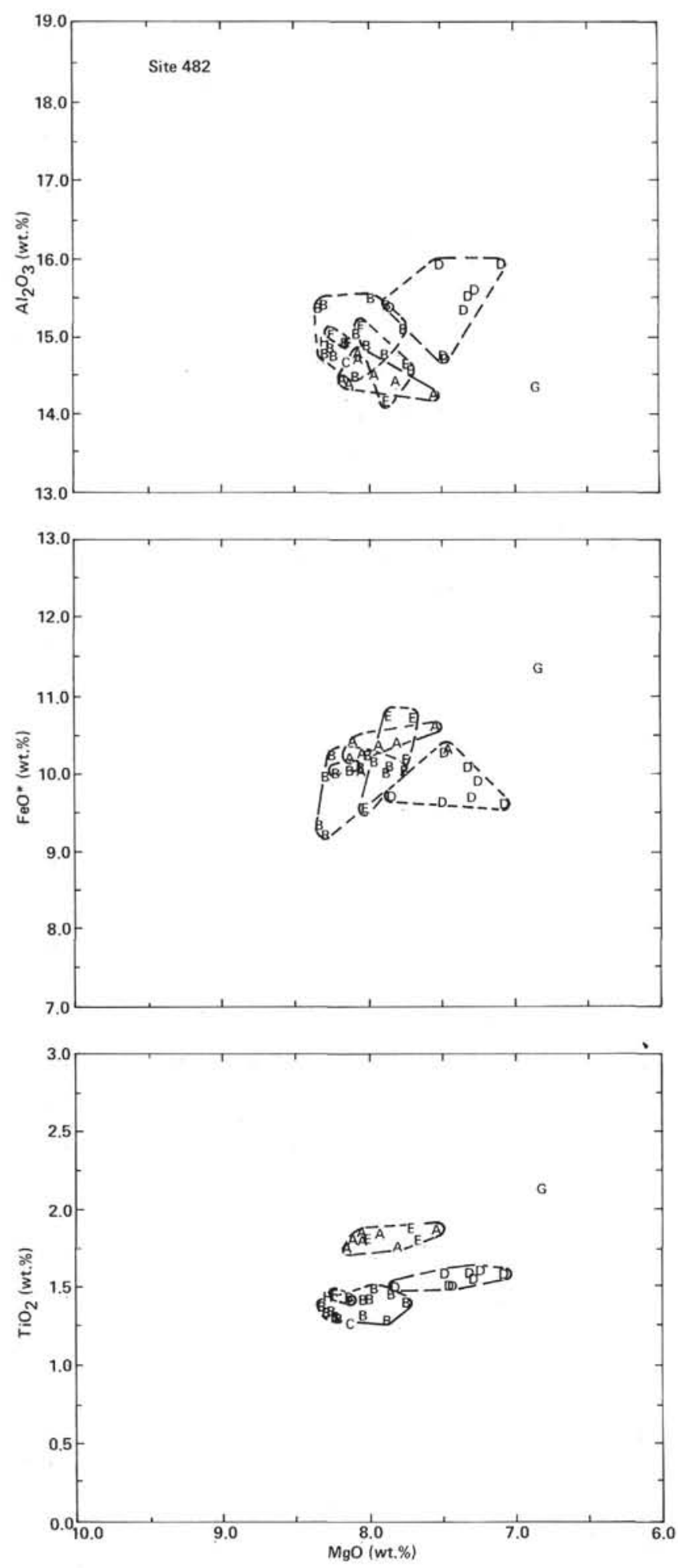

Figure 3. $\mathrm{Al}_{2} \mathrm{O}_{3}, \mathrm{FeO}^{*}$, and $\mathrm{TiO}_{2}$ versus $\mathrm{MgO}$ for Site 482. (Dashed lines indicate chemical types. Types A, B, C, E, and F are sparsely phyric or aphyric; Type D is moderately plagioclase-phyric.)

tallization, variable partial melting, and magma mixing in determining the overall chemical variation at these sites.

Variation diagrams for $\mathrm{Ti} / \mathrm{Zr}, \mathrm{Y} / \mathrm{Zr}, \mathrm{Sr} / \mathrm{Zr}$, and $\mathrm{Ni} /$ $\mathrm{Cr}$ are given in Figures 6 to 9 for all three sites. The data are labelled according to chemical type.
1) $\mathrm{Ti} / \mathrm{Zr}$ : $\mathrm{Ti}$ and $\mathrm{Zr}$ predictably show a positive covariance (Fig. 6), with the exception of Site 485 Type I which may reflect minor fractionation of magnetite. $\mathrm{The} \mathrm{Ti} / \mathrm{Zr}$ ratios appear uniform for Site 482 and 485 basalts but are distinctly variable for Site 483 and appear to depend on chemical type. The "upper" massive types (D, B, and C) show between-type variation, but the "lower" massive ( $\mathrm{H}$ and $\mathrm{K})$ and pillow $(\mathrm{F}, \mathrm{I}, \mathrm{J}, \mathrm{L}$, and $\mathrm{M})$ types can be divided into at least two series: namely, H-F and K-L-M-J-I, respectively. Discrepancies between the series are not compatible with simple fractionation or single-stage mixing models and are tentatively attributed to differences in source composition and/or the degree of partial melting.

2) $\mathrm{Y} / \mathrm{Zr}$ : Y variation for Site 482 (Fig. 7) distinguishes Types B, F, and D from Types A and E, which overlap. The Site 483 data show similar patterns in which the high $\mathrm{Zr}$ types (F, H, I, J, K, L, and M), which are interlayered "lower" massive and pillow sequences, are distinct from the low- $\mathrm{Zr}$ types (B, C, and D), the "upper" massive units. A similar pattern is also observed for Site 485 despite complex variation in Cooling Unit 9 (Type I). Cooling Unit 5 may reflect anomalous $\mathrm{Y} / \mathrm{Zr}$ ratios. As noted above, the basalts at all sites reflect increasing $\mathrm{Y} / \mathrm{Zr}$ ratios with increasing $\mathrm{Zr}$ content. This is best observed in the Site 483 data. However, no definitive between-site source differences are confirmed. Further low- $\mathrm{K}_{\mathrm{d}}$ element data are needed to test for this possibility.

3) $\mathrm{Sr} / \mathrm{Zr}$ : The variation of $\mathrm{Sr}$ at all of the sites (Fig. 8) corresponds closely to that observed for $\mathrm{Al}_{2} \mathrm{O}_{3}$ (Figs. 3-5) and is consistent with the inferred effects of plagioclase. Type D at Site $\mathbf{4 8 2}$ is plagioclase-phyric and shows $\mathrm{Sr}$ enrichment compared to aphyric Types A and E. Types $\mathrm{B}$ and $\mathrm{F}$ are unlikely to be related to Types $\mathrm{A}$ and E by simple fractionation unless olivine control was dominant or plagioclase was accumulated during the removal of the mafic phases. The variation at Site 483 shows similar patterns, but an apparent contradiction exists between the $\mathrm{Sr}$ enrichment in Chemical Types D, $\mathrm{H}$, and $\mathrm{K}$ (and major element mass balance requirements; see Table 6) and their aphyric to very sparsely phyric petrography, which shows no evidence of significant plagioclase accumulation. Major element mass balance considerations and $\mathrm{Sr}$ variation imply concurrent accumulation of plagioclase with mafic phase fractionation. The pillow sequences making up Chemical Types $\mathrm{F}, \mathrm{J}, \mathrm{L}$, and $\mathrm{M}$ also reflect similar mass balance relations, although the required enrichment in plagioclase is significantly less (Fig. 8; Table 6).

Sr variation in the Site 485 basalts is somewhat more erratic but loosely resembles that at the other sites. Cooling Units $1(\mathrm{~A}), 8(\mathrm{H}), 10(\mathrm{~J})$, and subunits of 9(I) reflect steep $\mathrm{Sr} / \mathrm{Zr}$ trends similar to that shown by Type D at Site 483. However, some of these units (8-10) reflect modal plagioclase indicative of accumulation.

4. $\mathrm{Ni} / \mathrm{Cr}: \mathrm{Ni} / \mathrm{Cr}$ ratios are insensitive to the fractionation and accumulation of plagioclase; their variation reflects the crystallization and redistribution of mafic phases and, possibly, sulfides. The $\mathrm{Ni} / \mathrm{Cr}$ variation for each site appears to reflect at least two converging trends (Fig. 9). This feature represents the ma- 
Table 6. Least-squares fractionation solutions, with $\mathrm{Zr}$, $\mathrm{Ti}$, and $\mathrm{Sr}$ enrichment factors, for parent-derivative whole-rock pairs.

\begin{tabular}{|c|c|c|c|c|c|c|c|c|c|c|}
\hline $\begin{array}{c}\text { Parent } \\
\text { Sample } \\
\text { (interval in } \mathrm{cm} \text { ) }\end{array}$ & C.T. ${ }^{a}$ & $\begin{array}{c}\text { Derivative } \\
\text { Sample } \\
\text { (interval in } \mathrm{cm} \text { ) }\end{array}$ & C. $T^{a}$ & $\begin{array}{l}\text { Olivine } \\
\left(\omega_{0)}\right)\end{array}$ & $\begin{array}{l}\text { Plagioclase } \\
\left(\sigma^{\circ}\right)\end{array}$ & $\begin{array}{c}\text { Clinopyroxene } \\
\left(\tilde{(}_{0}\right)\end{array}$ & $\Sigma R^{2}$ & [Zr]e & {$[\mathrm{Ti}]_{\mathrm{e}}$} & [Srle \\
\hline $482 \mathrm{~B}-22-3,103-107$ & D & $482 \mathrm{~B}-22-2,130-136$ & D & -1.72 & -0.90 & -2.92 & 0.08 & 1.04 & 1.05 & 0.97 \\
\hline $482 \mathrm{D}-10-1,106-109$ & $\mathrm{~F}$ & $482 \mathrm{D}-10-2,70-72$ & E & -3.43 & -12.43 & -7.65 & 0.04 & 1.37 & 1.27 & 1.15 \\
\hline $483 \mathrm{~B}-8 \cdot 1,55-61$ & D & $483 \mathrm{~B}-8-1,29-35$ & D & -1.45 & +5.63 & -0.62 & 0.12 & 0.97 & 0.96 & 1.31 \\
\hline $483-16-3,2-6$ & c & $483 \mathrm{~B}-7-3,2-6$ & c & -1.36 & -1.63 & -0.41 & 0.29 & 1.03 & 1.03 & 0.99 \\
\hline $483 \mathrm{~B}-4-1,26-30$ & B & $483-15-1,93-97$ & B & -0.91 & +1.71 & -0.01 & 0.15 & 0.99 & 0.99 & 1.20 \\
\hline $483 \cdot 24 \cdot 2,35-40$ & $\mathrm{H}$ & $483-25-2,61-66$ & $\mathrm{H}$ & -0.95 & +0.72 & -0.41 & 0.01 & 1.00 & 1.00 & 1.01 \\
\hline $483 \mathrm{~B}-20-2,50-59$ & $\mathrm{H}$ & $483 \mathrm{~B}-20-2,16-21$ & $\mathrm{H}$ & -0.51 & +5.55 & -0.20 & 0.24 & 0.96 & 0.95 & 1.02 \\
\hline $483 \mathrm{~B}-28-3,37-40$ & $\mathrm{~K}$ & $483 \mathrm{~B}-27-1,12-18$ & $\mathrm{~K}$ & -3.63 & -2.16 & -8.55 & 0.12 & 1.14 & 1.13 & 1.04 \\
\hline 483B-29-1, $10-15$ & $\hat{\mathrm{k}}$ & $483 \mathrm{~B}-25-2,22-28$ & $\hat{\mathrm{K}}$ & -2.97 & -5.52 & -10.40 & 0.10 & 1.16 & 1.19 & i.13 \\
\hline $483 \mathrm{~B}-26-2,126-137$ & $\ddot{k}$ & $483 \mathrm{~B}-27-1,114-119$ & $\mathrm{~K}$ & -3.81 & -1.91 & -0.11 & 0.17 & 1.03 & 1.06 & 1.02 \\
\hline $485 \mathrm{~A}-11-3,68-73$ & $\mathrm{~A}$ & $485 \mathrm{~A}-11-3,116-121$ & A & -3.39 & +1.63 & -0.39 & 0.06 & 1.01 & 1.02 & 1.70 \\
\hline $485 \mathrm{~A}-11-3,68-73$ & A & $485 \mathrm{~A}-11-3,57-60$ & A & -5.33 & +0.31 & -8.48 & 0.01 & 1.08 & 1.12 & 1.68 \\
\hline $485 \mathrm{~A}-13-1,110-117$ & $\hat{\mathrm{E}}$ & $485 \mathrm{~A}-13-1,11-8$ & $\hat{E}$ & -1.29 & +2.31 & -0.40 & 0.18 & 0.99 & 0.99 & 0.95 \\
\hline $485 \mathrm{~A}-25-1,117-143$ & $\mathrm{H}$ & 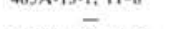 & & -2.42 & -4.75 & -1.65 & 0.07 & 1.07 & 1.09 & 1.01 \\
\hline $485 \mathrm{~A}-32-3,47-51$ & 1 & $485 \mathrm{~A}-32-6,52-57$ & 1 & -2.38 & -6.17 & -2.63 & 0.08 & 1.14 & 1.12 & 0.99 \\
\hline $485 \mathrm{~A}-33-2,26-33$ & $i$ & $485 \mathrm{~A}-33-2,88-94$ & i & -0.96 & -0.60 & $-1.1 ?$ & 0.10 & 1.10 & 1.02 & 1.04 \\
\hline $485 \mathrm{~A}-38-3,18-23$ & L & $485 A-38-6,58-63$ & $\mathrm{i}$ & -2.72 & -3.35 & -0.14 & 0.05 & 1.08 & 1.07 & 0.99 \\
\hline
\end{tabular}

jor distinction between Types B and F at Site 482, which are otherwise indistinguishable (Fig. 3). At Site 483, Types F, H, I, J, K, L, and M (the "lower" massive and pillow flows) together with Type D (from the upper massive flows) correspond to the high $\mathrm{Ni} / \mathrm{Cr}$ trend, while Types $\mathrm{C}$ and $\mathrm{B}$ (also from the upper massive flows) belong to the lower $\mathrm{Ni} / \mathrm{Cr}$ trend. None of the Site 485 types displays a low $\mathrm{Ni} / \mathrm{Cr}$ ratio, but Type $\mathrm{J}$ is intermediate between the two main trends that characterize the other sites. As with other element variation trends, the $\mathrm{Ni} / \mathrm{Cr}$ pattern for Cooling Unit 10 (Type I) is complex, in contrast to most of the other cooling units at Site 485 .

In summary, each chemical type appears to represent an individual fractionation trend superimposed on one or several preexisting "liquid"-type trend(s). Several fractionation "series" consisting of Types F-K, J-K-L, and $\mathrm{M}-$ ? are tentatively inferred from the $\mathrm{Ti} / \mathrm{Zr}$ variations at Site 483 and appear to be consistent with the $\mathrm{Sr}$, $\mathrm{Ni}$, and $\mathrm{Cr}$ variation as well (Fig. 10). Considered stratigraphically (Fig. 2), these may reflect a cyclic eruptive pattern of massive and pillowed lavas (in that order). As indicated by the $\mathrm{Y} / \mathrm{Zr}$ variation, these chemical types are distinct from the "upper" massive units (Types B, C, and D) with Type D showing the most obvious difference. We note again the apparent conflict between mass balance requirements for plagioclase accumulation and absence or paucity of plagioclase as a phenocryst phase.

\section{SUMMARY AND DISCUSSION}

The major and trace element chemistry of the basalts from these sites reveals complex variation patterns which clearly indicate the influence of several types and hierarchies of petrogenetic processes. Fractional crystallization is obviously an important contributor to the variation observed. However, the diversity of the trends at any given site appears inconsistent with single-stage, closed-system fractionation models (Fig. 10). Mass balance models based on least-squares calculations should not be overemphasized in view of analytical (and other) uncertainties. Contradictions of interpretations based on trace and major elements (e.g., Samples 485A-11-3, $68-73 \mathrm{~cm}$ and $116-121 \mathrm{~cm}$, in which an increase in $\mathrm{Ni}$ from 208 to 233 appears to correspond to the removal of
$3.39 \%$ olivine) may result from quantitative rather than qualitative inaccuracies in the solution. In general, the mass balance models for single eruptive units suggest that the compositions of these units are the result of repeated episodes of phenocryst redistribution in magma batches derived from earlier, probably more deep-seated, fractionation episodes. Mass balance requirements for several of the massive units indicate that plagioclase accumulation was accompanied by fractional removal of olivine and clinopyroxene. The petrography of some units is consistent with this interpretation, as reflected by significant phenocryst plagioclase associated with little or no mafic phases. However, plagioclase rarely exceeds 5 modal percent, and in several notable examples, is altogether absent (e.g., Types D, K, and H at Site 483 and Types A and I at Site 485).

The accumulation of plagioclase may occur under static or dynamic conditions. During the rise of the magma, cotectic crystallization of olivine, plagioclase, and clinopyroxene may result in gravitational separation of the mafic phases and plagioclase, the latter being retained in suspension (e.g., Fujii and Kushiro, 1977; Kushiro, 1980). Alternatively, plagioclase may accumulate by flotation in a stable crystallizing magma body, a process that Flower (1980) suggests may be favored by higher pressure fractionation regimes $(>\mathrm{ca} .5 \mathrm{~kb})$ in view of the relative increase in liquid tholeiite density (Fujii and Kushiro, 1977). In either case, a magma in which plagioclase is present as a cumulate phase will diverge compositionally from "liquid-line" trends. To explain aphyric magmas with the compositional character of cumulates, a mechanism is required for redissolving phenocryst phases such that a totally fluid magma is erupted at the surface. Such explanations are probably best sought in terms of basaltic phase equilibria. Two critical effects are brought to mind: (1) the effect of variable pressure on the stability of solid phases (polybaric fractionation) (O'Hara, 1968; Presnall et al., 1979; Bender et al., 1978) and (2) superheating resulting from proximity to or hybridization with hot primitive magma (e.g., Goode, 1977; Elthon, 1981).

Possible scenarios combining polybaric fractionation regimes and magma mixing could thus explain the principal characteristics of the basaltic variation observed 

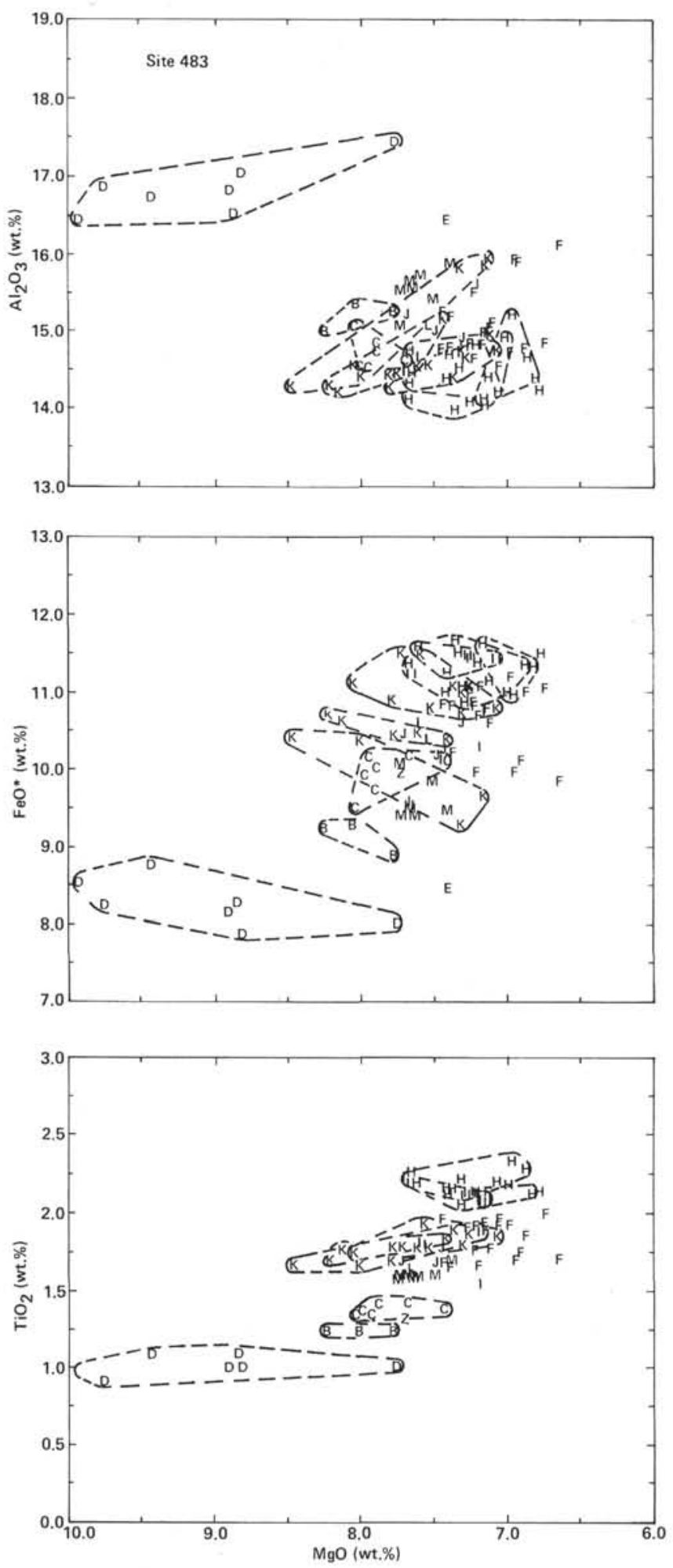

Figure 4. $\mathrm{Al}_{2} \mathrm{O}_{3}, \mathrm{FeO}^{*}$, and $\mathrm{TiO}_{2}$ versus $\mathrm{MgO}$ for Site 483. Dashed lines indicate "upper" and "lower" massive units (Chemical Types B, C, and D and Types $\mathrm{H}$ and $\mathrm{K}$, respectively). Pillow lavas consist of Types F, I, J, L, and M; Types B, C, D, and K are aphyric or very sparsely phyric; Type $\mathrm{H}$ and pillow basalt sequences are generally more phyric. (Note distinctive massive unit trends compared to overall trends of $\mathrm{FeO}^{*}$ and $\mathrm{TiO}_{2}$-enrichment and $\mathrm{Al}_{2} \mathrm{O}_{3}$-depletion.)
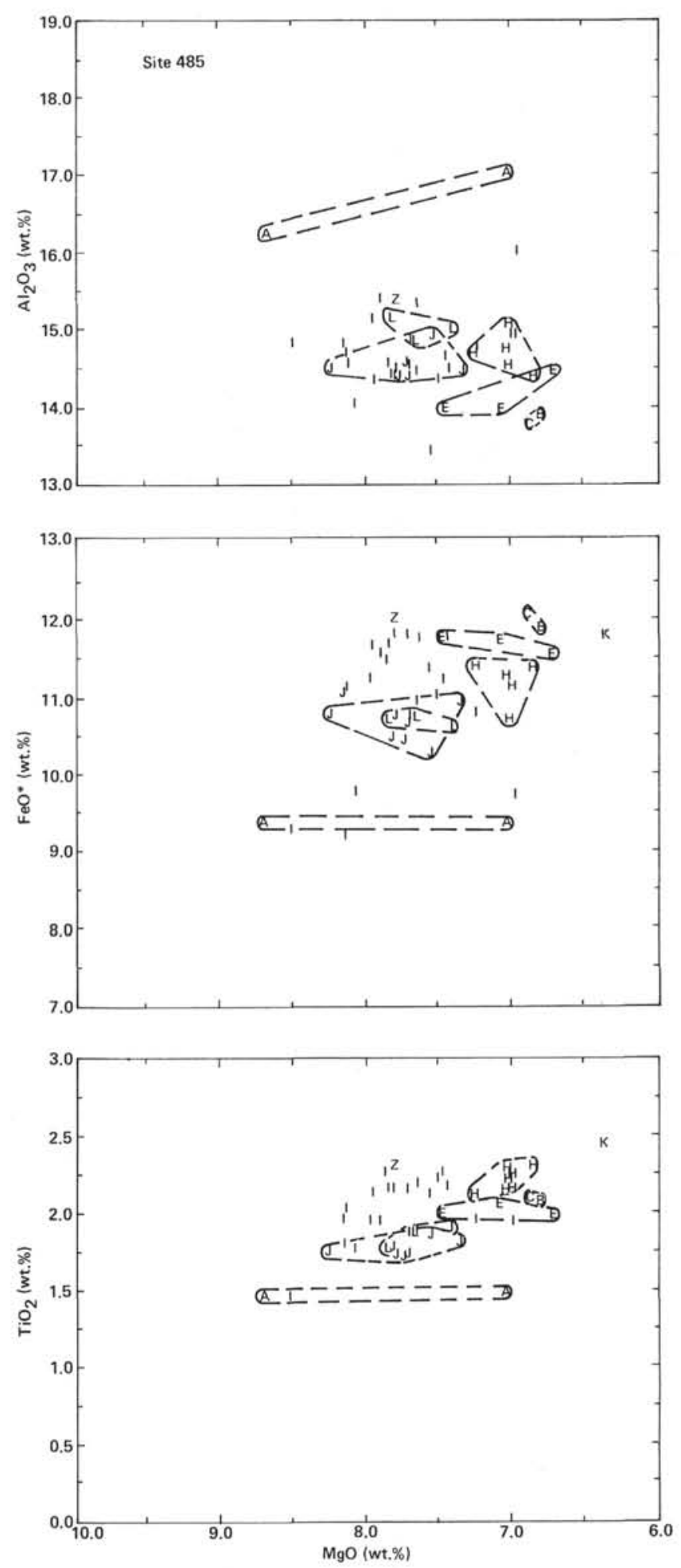

Figure 5. $\mathrm{Al}_{2} \mathrm{O}_{3}, \mathrm{FeO}^{*}$, and $\mathrm{TiO}_{2}$ versus $\mathrm{MgO}$ for Site 485. (Dashed lines indicate chemical types corresponding to lithologic units. Types A, B, and C correspond to Unit 1; Type I corresponds to Unit 5, which is believed to result from multiple eruption and/or intrusion. Note similarity of intra-unit variation to that observed in massive units at Site 483.) 

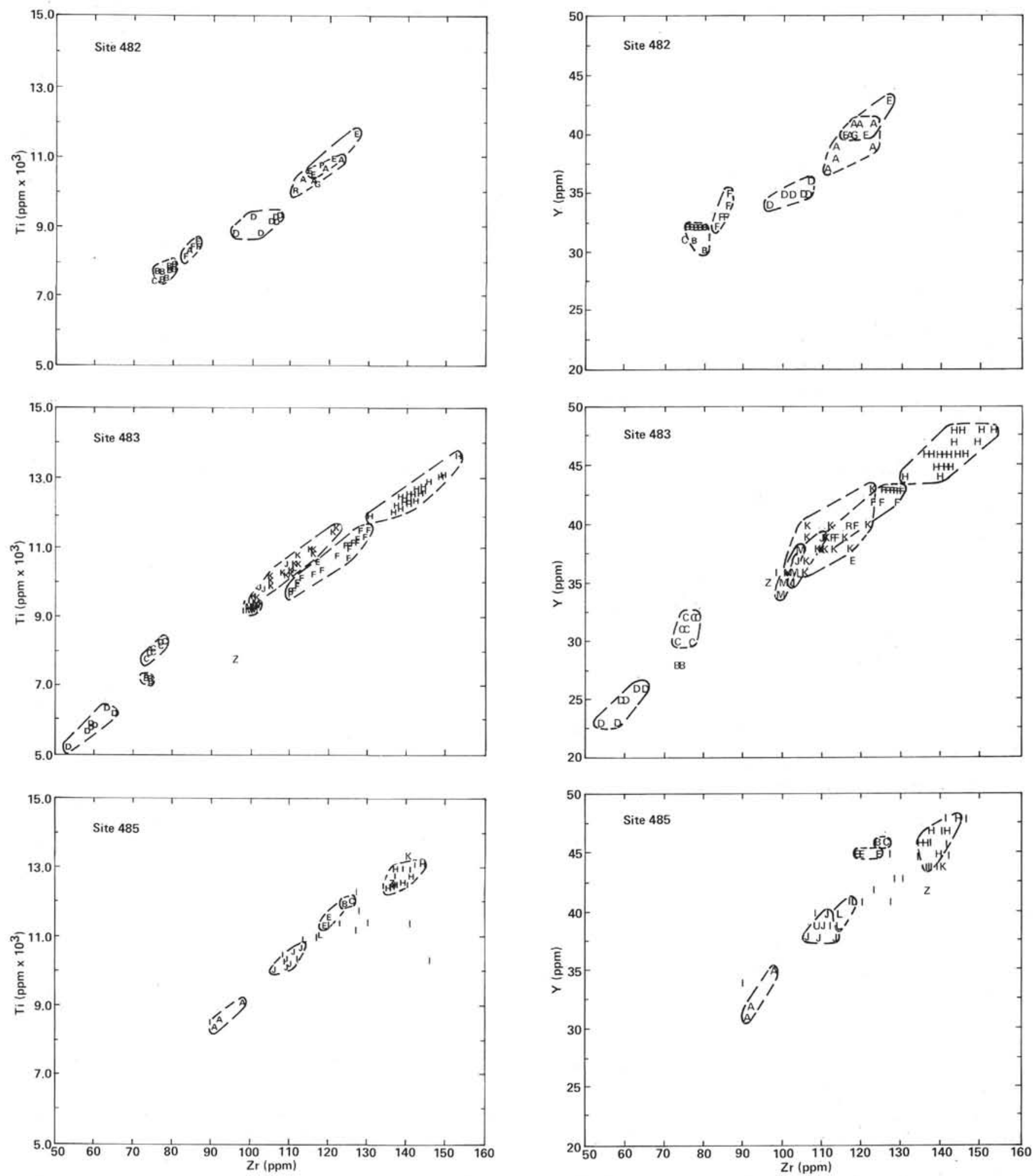

Figure 6. Ti versus $\mathrm{Zr}$ for Sites 482,483 , and 485 . (Dashed lines indicate chemical types. Note distinctive $\mathrm{Ti} / \mathrm{Zr}$ ratios for chemical types.)

Figure 7. Y versus $\mathrm{Zr}$ for Sites 482, 483, and 485. (Dashed lines indicate chemical types. Note approximately continuous betweentype variation and the increase in $\mathrm{Zr} / \mathrm{Y}$ ratio with increasing $\mathrm{Zr}$ content.) 

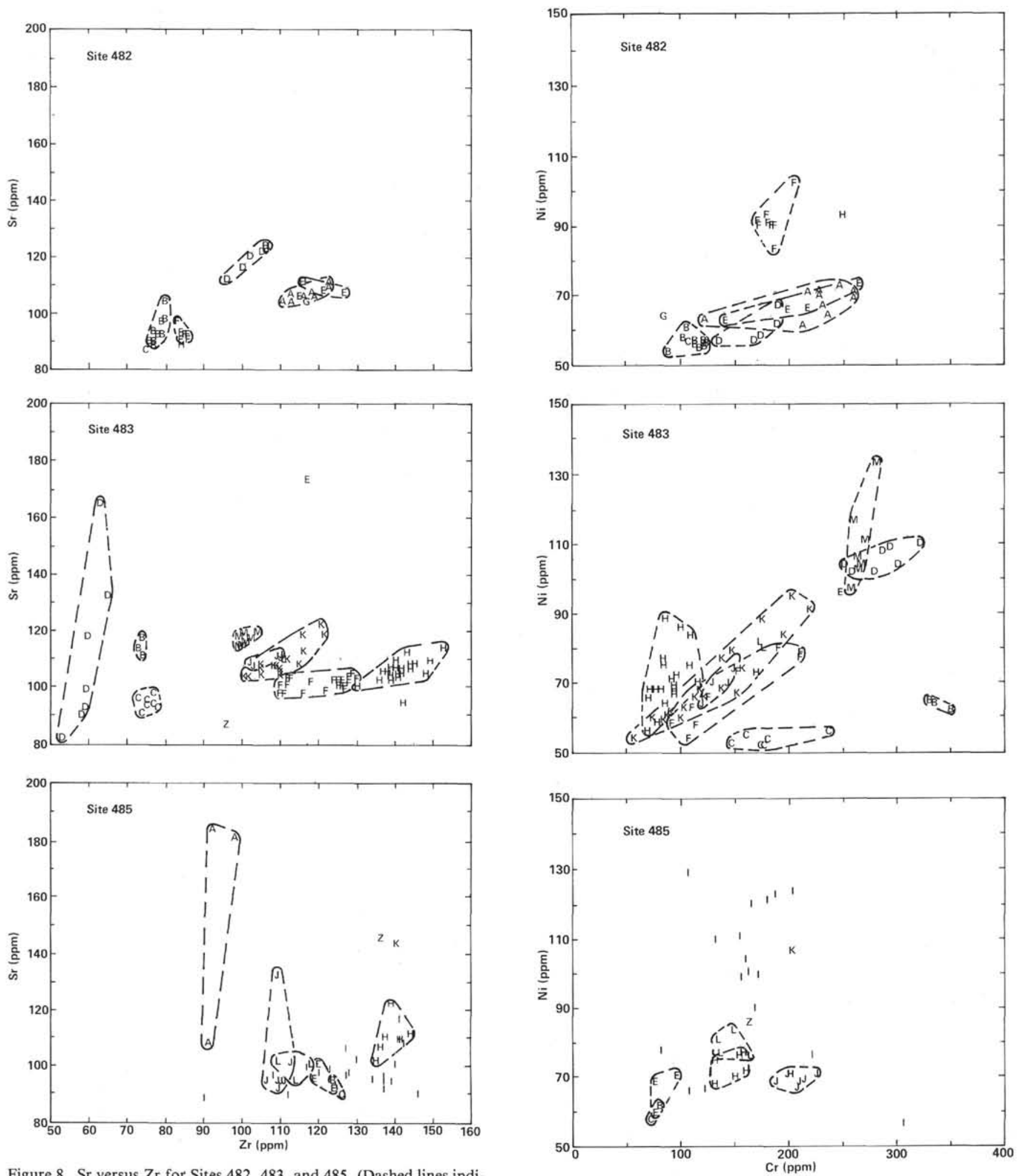

Figure 8. Sr versus $\mathrm{Zr}$ for Sites 482, 483, and 485. (Dashed lines indicate chemical types. Note discrepancy between intraunit and overall variation trends [cf. major elements, especially $\mathrm{Al}_{2} \mathrm{O}_{3}$ ]. This results partly from plagioclase accumulation.)

Figure 9. Ni versus $\mathrm{Cr}$ for Sites 482,483 , and 485 . (Dashed lines indicate chemical types. Note high and low $\mathrm{Ni} / \mathrm{Cr}$ trends at Sites 482 and 483 and the dispersion of values for Site 485 Type I.) 


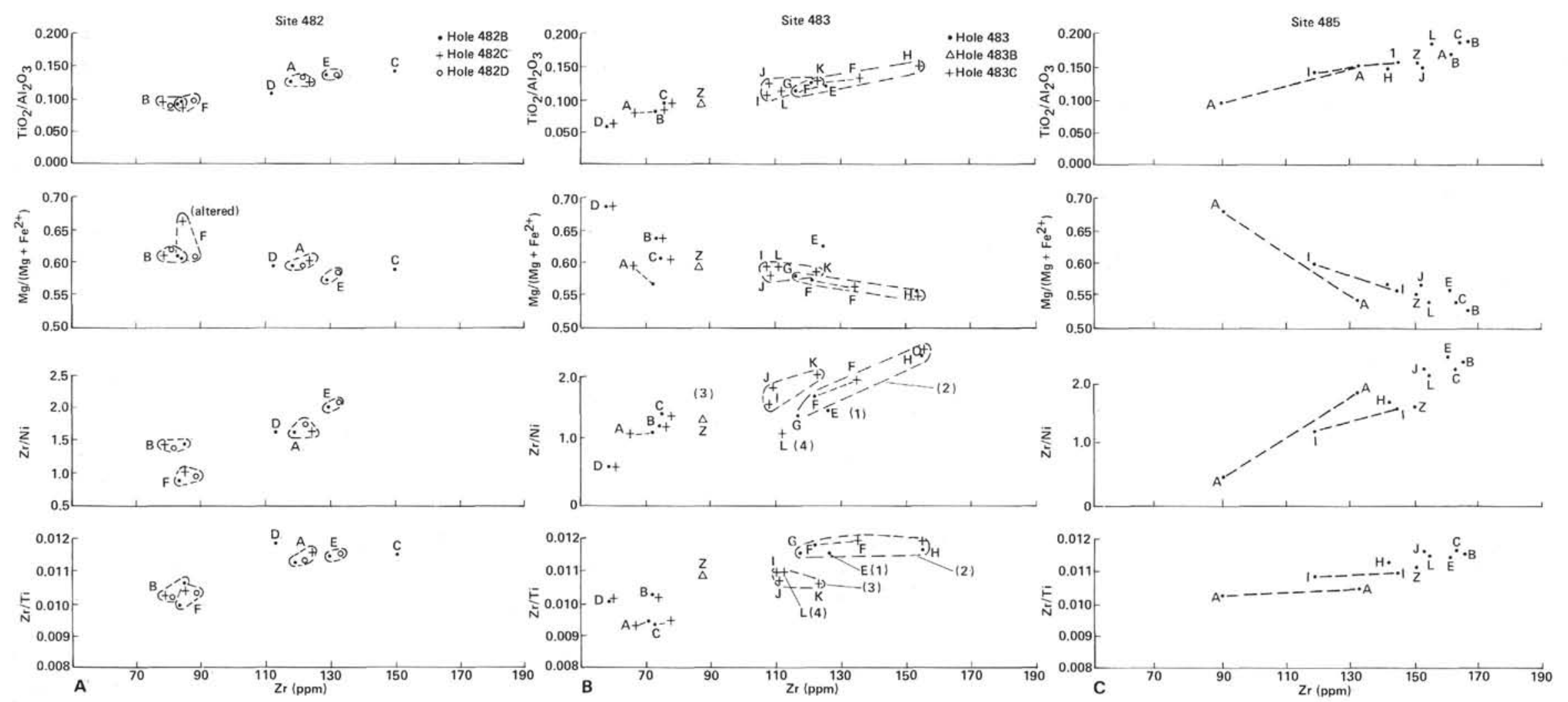

Figure 10. A. Chemical type averages for $\mathrm{TiO}_{2} / \mathrm{Al}_{2} \mathrm{O}_{3}, \mathrm{Mg} /\left(\mathrm{Mg}+\mathrm{Fe}^{2+}\right), \mathrm{Zr} / \mathrm{Ni}$, and $\mathrm{Zr} / \mathrm{Ti}$ versus $\mathrm{Zr}$ for holes at Site 482 . B. $\mathrm{Chemical} \mathrm{type} \mathrm{averages} \mathrm{for} \mathrm{TiO} / \mathrm{Al}{ }_{2} \mathrm{O}_{3}, \mathrm{Mg} /\left(\mathrm{Mg}+\mathrm{Fe}{ }^{2+}\right), \mathrm{Zr} / \mathrm{Ni}$, and $\mathrm{Zr} / \mathrm{Ti}$ versus $\mathrm{Zr}$ for holes at Site 483 . (Dashed lines indicate massive/pillow unit cyclic sequences.) $\mathrm{C}$. Chemical type averages for TiO $/ \mathrm{Al}_{2} \mathrm{O}_{3}$, $\mathrm{Mg} /\left(\mathrm{Mg}+\mathrm{Fe}{ }^{2+}\right), \mathrm{Zr} / \mathrm{Ni}$, and $\mathrm{Zr} / \mathrm{Ti}$ versus $\mathrm{Zr}$ for Site 485. Averages based on composite data sets (this work and shipboard data). (A...... $\mathrm{A}^{\prime}$ and $\mathrm{I}$..... $\mathrm{I}^{\prime}$ indicate low and high $\mathrm{MgO}$ subtype averages.) 
along this part of the East Pacific Rise. It is noted that the plagioclase-cumulate trends observed in the Leg 65 basalts (cryptic or otherwise) are considerably less well developed than those commonly observed in Atlantic basalts (e.g., from Sites 332, 395, 396, 417, and 418) where, in most cases, plagioclase-enrichment trends are marked by strongly plagioclase-phyric lithologies (Flower, 1980). It should also be noted that the variation patterns shown in Figures 3-9 suggest that mixing was not of the "open-system"' type (e.g., O'Hara, 1977; 1981), but occurred, together with fractional crystallization and phenocryst accumulation, during discrete episodes.

In general, the geometric and temporal character of magmatic accretion processes in the southern Gulf of California appears to be intermediate between the processes observed along superfast sections of the East $\mathrm{Pa}$ cific Rise and those along the slow-spreading Mid-Atlantic Ridge. The magma fractionation systems beneath the Gulf of California axis resemble those of the MidAtlantic Ridge in being transient, possibly polybaric and giving rise to plagioclase-cumulate magmas. In contrast, the emplacement of magma appears to be under relatively stable tectonic conditions more typical of the fastspreading environment.

\section{ACKNOWLEDGMENTS}

Thanks are extended to the captain and crew of the D/V Glomar Challenger and to the staff of the Deep Sea Drilling Project for ensuring the success of Leg 65 . Funding of this research was provided by Deutsche Forschungsgemeinschaft (West Germany), the Smithsonian Institution (Washington, D.C.), the National Research Council (Canada), and the Natural Environment Research Council (U.K.)

\section{REFERENCES}

Bender, J. F., Hodges, F. N., and Bence, A. E., 1978. Petrogenesis of basalts from the project FAMOUS area: Experimental study from 0 to 15 kilobars. Earth Planet. Sci. Lett., 41:277-302.

Bryan, W. B., Thompson, G., and Frey, F. A., 1979. Petrologic character of the Atlantic crust from DSDP and IPOD drill sites. In Talwani, M., Harrison, C. G., and Hayes, D. E. (Eds.), Sec. Maurice Ewing Series: Washington (Am. Geophys. Union), Spec. Publ. 1:237-284.

Byerly, G. R., and Wright, T. L., 1978. Origin of major element chemical trends in DSDP Leg 37 basalts, Mid-Atlantic Ridge. J. Volcanol. Geotherm. Res., 3:229-279.

CYAMEX Scientific Group, in press. First manned submersible dives on the East Pacific Rise, $21^{\circ} \mathrm{N}$. (Project RITA): General results. Mar. Geophys. Res.

Elthon, D., 1979. High magnesia liquids as the parental magma for ocean floor basalts. Nature, 278:514-518.

,1981. One atmosphere phase equilibria of basalts from the Tortuga ophiolite complex, with implications for magma chamber processes. The Generation of Oceanic Lithosphere (Chapman Conference, Airlie House, Virginia), p. 10.

Flower, M. F. J., 1980. Accumulation of calcic plagioclase in oceanridge tholeiite: An indication of spreading rate?. Nature, 287: 530-532.

1981. A thermal-kinematic model for ocean-ridge magma fractionation. J. Geol. Soc. Lond.: 138.

Flower, M. F. J., and Robinson, P. T., 1978. Evolution of the FAMOUS ocean ridge segment: Evidence from submarine and deep sea drilling investigations. In Talwani, M., Harrison, C. G., and Hayes, D. E. (Eds.), Deep Drilling Results in the Atlantic Ocean: Ocean Crust. Sec. Maurice Ewing Series: Washington (Am. Geophys. Union), pp. 314-330.

1981a. Basement drilling in the Western Atlantic. 1. Magma fractionation and its relation to eruptive chronology. J. Geophys. Res., 86:6273-6298.

1981b. Basement drilling in the Western Atlantic. 2. A synthesis of construction processes at the Cretaceous ridge axis. $J$. Geophys. Res., 86:6299-6309.

Flower, M. F. J., Robinson, P. T., Ohnmacht, W., Marriner, G., and Schmincke, H.-U., 1980. Lithologic and chemical stratigraphy at DSDP Sites 417 and 418. In Donnelly, T., Francheteau, J., Bryan, W., Robinson, P., Flower, M., Salisbury, M., et al., Init. Repts. DSDP, 51, 52, 53, Pt. 2: Washington (U.S. Govt. Printing Office), 939-956.

Flower, M. F. J., Robinson, P. T.,, Schmincke, H.-U., and Ohnmacht, W., 1977. Magma fractionation systems beneath the MidAtlantic Ridge near 36-37 ${ }^{\circ}$. Contr. Mineral. Petrol., 64:167-195.

Fujii, T., and Kushiro, I., 1977. Density, viscosity and compressibility of basaltic liquid at high pressure. Dir. Geophys. Lab., Carnegie Inst. Washington Yearbook, 76:419-424.

Francheteau, J., Juteau, T., and Rangin, C., 1979. Basaltic pillars in collapsed lava-pools on the deep ocean floor. Nature, 281: 209-211.

Goode, A. D. T., 1977. Flotation and remelting of plagioclase in the Kalka Intrusion, central Australia: Petrological implications for anorthosite genesis. Earth Planet. Sci. Lett., 34:379-397.

Huppert, H. E., and Sparks, R. S. J., 1980. Restrictions on the compositions of mid-ocean ridge basalts: A fluid dynamical investigation. Nature, 286:46-48.

Kastens, K. A., Macdonald, K. C., Becker, K., and Crane, K., 1979. The Tamayo transform fault in the mouth of the Gulf of California. Mar. Geophys. Rev, 4:129-131.

Kushiro, 1., 1980. Viscosity, density, and structure of silicate melts at high pressures, and their petrological applications. In Hargraves, R. B. (Ed.), Physics of Magmatic Processes: Princeton (Princeton University Press), pp. 93-120.

Lewis, B. T. R., 1979. Periodicities in volcanism and longitudinal magma flow on the East Pacific Rise at $23^{\circ} \mathrm{N}$, Geophys. Res. Lett., 6:53-756.

O'Hara, M. J., 1968. The bearing of phase equilibrium studies in synthetic and natural systems on the origin and evolution of basic and ultrabasic rocks. Earth Sci. Rev., 4:69-133.

, 1977. Geochemical evolution during fractional crystallization of a periodically refilled magma chamber. Nature, 266: 503-507.

1981 Geochemical evolution in an advancing, periodically replenished, periodically tapped, continuously fractionated magma chamber. J. Geol. Soc. Lond., 138.

Presnall, D. C., Dixon, J. G., O’Donnell, T., and Dixon, S. A., 1979. Generation of mid-ocean ridge tholeiite. J. Petrol., 20:3-35.

Reid, 1., Orcutt, J. A., and Prothero, W. A., 1977. Seismic evidence for a narrow zone of partial melting underlying the East Pacific Rise at $21^{\circ}$ N. Bull. Geol. Soc. Am., 88:678-682.

APPENDIX A

Analyzed Whole-Rock Samples

\begin{tabular}{cccc}
\hline Sample & Lithologic & Cooling & Chemical \\
Unit & Unit & Type \\
\hline
\end{tabular}

Hole 482B

$13-1,99-104$
$14-1,91-94$
$14-1,132-137$
$14-3,2-6$
$14-4,16-20$
$15-1,12-16$
$16-4,100-104$
$20-1,27-30$
$20-3,144-147$
$21-2,19-23$
$21-3,58-63$
$22-2,67-72$
$22-2,130-136$
$22-3,103-107$

1

1 (massive)

5 (massive)

6

8 (massive)

8

9 (massive)

11 (massive)

13 (massive)

15 (massive)

15

16 (massive)

16

16

16

A
F
F
F
F
F
B
B
D
D
D
D
D
D


Appendix A. (Continued).

\begin{tabular}{cccc}
\hline $\begin{array}{c}\text { Sample } \\
\text { (interval in cm) }\end{array}$ & $\begin{array}{c}\text { Lithologic } \\
\text { Unit }\end{array}$ & $\begin{array}{c}\text { Cooling } \\
\text { Unit }\end{array}$ & $\begin{array}{c}\text { Chemical } \\
\text { Type }\end{array}$ \\
\hline
\end{tabular}

Hole 482B

$22-4,97-102$

$$
16
$$

17 (massive)

D

Hole $482 \mathrm{C}$

$$
\begin{aligned}
& 10-1,0-4 \\
& 10-1,9-12 \\
& 10-1,12-16 \\
& 10-3,11-14 \\
& 11-3,65-69 \\
& 12-1,92-118 \\
& 13-1,10-15 \\
& 14-1,13-18 \\
& 15-1,114-118
\end{aligned}
$$

$8 \quad 17$ (massive)

D

Hole 482D

$$
\begin{aligned}
& 8-1,24-29 \\
& 8-1,65-72 \\
& 10-1,106-109 \\
& 10-1,111-114 \\
& 10-2,70-72 \\
& 10-2,128-130 \\
& 10-3,25-27 \\
& 10-3,93-97 \\
& 12-1,28-32 \\
& 12-3,27-31 \\
& 13-1,49-51 \\
& 13-1,98-101 \\
& 13-1,128-134
\end{aligned}
$$

$\begin{array}{ll}1 & 1 \text { (massive) } \\ & 1 \\ & 1 \\ & 1 \\ & 2 \text { (massive) } \\ & 3 \text { (massive) } \\ & 4 \text { (massive) } \\ & 5 \text { (massive) } \\ & 7 \text { (massive) }\end{array}$

A
A
A
A
A
B
B
B
B

Hole $482 \mathrm{~F}$

$$
\text { 5-1, 133-139 }
$$

$11-1,125-128$

$\begin{array}{ll}1 & 1 \text { (massive) } \\ & 1 \\ 2 & 3 \text { (massive) } \\ & 3 \mathrm{a} \\ & 3 \mathrm{a} \\ & 3 \mathrm{a} \\ & 3 \mathrm{a} \\ & 5 \text { (pillow?) } \\ & 10 \text { (pillow?) } \\ & 12 \text { (massive) } \\ & 14 \text { (massive) } \\ & 14 \\ & 14\end{array}$

Hole 483

$\begin{array}{lll}14-1,58-68 & 2 & 2 \mathrm{a} \text { (massive) } \\ 15-1,93-97 & & 2 \mathrm{~b} \\ 16-2,64-70 & 3 & 3 \text { (massive) } \\ 16-2,99-100 & & 3 \\ 16-3,2-6 & & 3 \\ 17-2,13-17 & 4 & 4 \text { (massive) } \\ 17-2,17-21 & & 4 \\ 18-4,131-134 & 5 & 5 \text { (massive) } \\ 21-1,49-55 & 6 & 13 \text { (pillow) } \\ 21-1,57-61 & & 13 \\ 21-3,6-9 & & 16 \text { (pillow) } \\ 22-2,68-72 & & 23 \text { (pillow) } \\ 22-2,74-77 & & 23 \\ 22-2,85-88 & & 23 \\ 22-2,88-93 & & 23 \\ 22-2,94-99 & & 23 \\ 23-1,10-16 & & 36 \text { (pillow) } \\ 24-1,52-57 & 7 \text { (a) } & 40 \text { (massive) } \\ 24-2,35-40 & & 41 \text { (massive) } \\ 25-2,61-66 & & 44 \text { (massive) } \\ 26-1,59-63 & 7 \text { (b) } & 45 \text { (massive) } \\ 26-2,40-44 & & 46 \text { (massive) } \\ 26-3,124-130 & & 46\end{array}$

B
B
C
C
C
D
D
E
F
F
F
F
F
F
F
F
F
H
H
H
H
H
H

Hole 483B

$$
\begin{aligned}
& 4-1,26-30 \\
& 7-1,7-12 \\
& 7-1,26-31 \\
& 7-2,56-61
\end{aligned}
$$$$
7-3,2-6
$$

Appendix A. (Continued).

\begin{tabular}{cccc}
\hline $\begin{array}{c}\text { Sample } \\
\text { (interval in cm) }\end{array}$ & $\begin{array}{c}\text { Lithologic } \\
\text { Unit }\end{array}$ & $\begin{array}{c}\text { Cooling } \\
\text { Unit }\end{array}$ & $\begin{array}{c}\text { Chemical } \\
\text { Type }\end{array}$ \\
\hline
\end{tabular}

Hole 482B (Cont.)

$8-1,29-35$
$8-1,55-61$

$8-1,120-126$

$8-2,121-126$

$8-7,2-20$

$12-1,92-95$

$13-1,38-44$

$13-1,60-64$

$13-1,60-64$

$13-1,66-67$

$13-1,66-67$

13-1, 74-79

13-1, 92-98

$13-1,108-119$

$13-1,115-122$

$13-1,115-122$

13-1, 124-131

$13-1,124-131$

$13-1,134-139$

$14-1,130-133$

$18-1,49-52$

$18-1,103-107$

$18-2,18-22$

$19-2,17-21$

19-2, 104-108

$19-3,8-12$

20-1, 14-19

20-1, 94-99

20-2, 9-14

20-2, 16-21

20-2, 25-30

20-2, 37-42

20-2, 50-59

20-2, 50-59

$20-2,50-59$

20-2, 142-146

21-2, 57-61

22-1, 26-32

22-2, 63-67

22-2, 77-82

$23-4,19-24$

25-2, 18-20

25-2, 22-28

25-2, 39-45

26-2, 42-47

26-2, 126-137

$27-1,12-18$

27-1, 114-119

27-2, 95-101

$27-3,94-98$

27-4, 74-80

28-1, 42-59

28-1, 123-128

28-2, 28-32

28-3, 37-40

29-1, 10-15

29-1, 26-31

29-1, 40-45

29-1, 55-57

29-1, 57-62

29-1, 57-62

$30-3,13-16$

$30-3,18-22$

$31-3,20-25$

$32-1,1-6$

32-1, 27-31

$32-1,44-50$
4

4 (massive)

4

4

4

5(a) 8 (pillow)

5(b) $\quad 12 / 23$ (pillow)

$12 / 23$

$12 / 23$

$13 / 24$ (pillow)

$13 / 24$ (pillow)

$13 / 24$

$13 / 24$

$13 / 24$

$13 / 24$

$13 / 24$

14/25 (pillow)

$14 / 25$

$14 / 25$

$21 / 32$ (pillow)

6(b) 24/40 (massive)

$24 / 40$

24/40

6(c) $25 / 42$ (massive)

6(d) 26/43 (massive)

$26 / 43$

$26 / 44$

$26 / 44$

$26 / 44$

$26 / 44$

$26 / 44$

$26 / 44$

$26 / 44$

$26 / 44$

$26 / 44$

7(a) $27 / 46$ (massive)

7(b) $\quad 31 / 50$ (pillow)

35/54 (pillow)

$39 / 58$ (pillow)

39/58 (pillow)

49/68 (pillow)

8(a) $\quad 57 / 77$ (massive)

58/78 (massive)

$58 / 78$

$58 / 80$

$58 / 81$

8(b) $\quad 59 / 83$ (massive)

$59 / 83$

$59 / 83$

$59 / 83$

$59 / 83$

8(c) $60 / 84$ (massive)

$60 / 84$

$60 / 84$

$60 / 84$

$60 / 85$

$60 / 85$

$60 / 85$

$60 / 85$

$60 / 85$

$60 / 85$

68/94 (pillow)

69/95 (pillow)

74/101 (pillow)

77/104 (pillow)

$77 / 104$

$77 / 104$ 
Appendix A. (Continued).

\begin{tabular}{cccc}
\hline $\begin{array}{c}\text { Sample } \\
\text { (interval in cm) }\end{array}$ & $\begin{array}{c}\text { Lithologic } \\
\text { Unit }\end{array}$ & $\begin{array}{c}\text { Cooling } \\
\text { Unit }\end{array}$ & $\begin{array}{c}\text { Chemical } \\
\text { Type }\end{array}$ \\
\hline
\end{tabular}

Hole 482B (Cont.)

$\begin{array}{lll}32-1,90-94 & 77 / 104 & \mathrm{M} \\ 32-1,90-94 & 77 / 104 & \mathrm{M} \\ 32-1,84-88 & 77 / 104 & \mathrm{M}\end{array}$

Hole $483 \mathrm{C}$

4-4, 58-66 $\quad 1 \quad 1$ (massive) $\quad \mathrm{Z}$

Hole 485A

\begin{tabular}{llll}
$11-3,57-60$ & 1 & $1 / 1$ (massive) & $\mathrm{A}$ \\
$11-3,68-73$ & & $1 / 1$ & $\mathrm{~A}$ \\
$11-3,116-121$ & & $1 / 1$ & $\mathrm{~A}$ \\
$12-1,10-16$ & & $2 / 3$ (massive) & $\mathrm{B}$ \\
$12-1,76-82$ & & $3 / 4$ (massive) & $\mathrm{C}$ \\
$12-1,114-120$ & & $5 / 6$ (massive) & $\mathrm{E}$ \\
$13-1,11-18$ & & $5 / 7$ & $\mathrm{E}$ \\
$13-1,110-117$ & & $5 / 7$ & $\mathrm{E}$ \\
$17-1,77-82$ & 2 & $6(?)$ & $\mathrm{Z}$ \\
$23-1,50-56$ & 4 & $8 / 12$ (pillow?) & $\mathrm{H}$ \\
$23-1,50-56$ & & $8 / 12$ & $\mathrm{H}$ \\
$23-2,16-22$ & & $8 / 12$ & $\mathrm{H}$ \\
$23-3,120-125$ & & $8 / 12$ & $\mathrm{H}$ \\
$25-1,117-143$ & & $8 / 14$ & $\mathrm{H}$ \\
$29-1,2-8$ & 5 & $9 / 16$ (massive) & $\mathrm{I}$ \\
$29-1,89-93$ & & $9 / 16$ & $\mathrm{I}$ \\
$29-2,143-148$ & & $9 / 16$ & $\mathrm{I}$ \\
$29-3,10-16$ & & $9 / 16$ & $\mathrm{I}$ \\
$30-2,96-101$ & & $9 / 19$ & $\mathrm{I}$ \\
\hline
\end{tabular}

Appendix A. (Continued).

\begin{tabular}{cccc}
\hline Sample & Lithologic \\
(interval in cm) & Unit & $\begin{array}{c}\text { Cooling } \\
\text { Unit }\end{array}$ & $\begin{array}{c}\text { Chemical } \\
\text { Type }\end{array}$ \\
\hline
\end{tabular}

Hole 485A (Cont.)

\begin{tabular}{|c|c|c|}
\hline $30-3,38-41$ & & $9 / 19$ \\
\hline $30-3,44-49$ & & $9 / 19$ \\
\hline $30-4,8-13$ & & $9 / 19$ \\
\hline $31-1,62-67$ & & $9 / 20$ \\
\hline $31-3,34-39$ & & $9 / 20$ \\
\hline $32-1,55-61$ & & $9 / 20$ \\
\hline $32-2,99-105$ & & $9 / 21$ \\
\hline $32-3,47-51$ & & $9 / 21$ \\
\hline $32-5,9-14$ & & $9 / 21$ \\
\hline $32-6,52-57$ & & $9 / 21$ \\
\hline $33-1,2-9$ & & $9 / 22$ \\
\hline $33-1,74-79$ & & $9 / 22$ \\
\hline $33-2,26-33$ & & $9 / 22$ \\
\hline $33-2,72-78$ & & $9 / 22$ \\
\hline $33-2,88-94$ & & $9 / 22$ \\
\hline $34-1,86-90$ & 6 & 10/23 (massive) \\
\hline $34-2,96-102$ & & $10 / 23$ \\
\hline $35-1,135-142$ & & $10 / 23$ \\
\hline $35-2,113-118$ & & $10 / 24$ \\
\hline $35-3,21-27$ & & $10 / 24$ \\
\hline $35-4,85-92$ & & $10 / 24$ \\
\hline $35-6,47-53$ & & $10 / 24$ \\
\hline $36-3,65-69$ & 7 & $11 / 25$ (massive) \\
\hline $38-3,18-23$ & 8 & $12 / 27$ (massive) \\
\hline $38-6,58-63$ & & $12 / 27$ \\
\hline $39-1,26-32$ & & $12 / 28$ \\
\hline $39-1,59-64$ & & $12 / 28$ \\
\hline
\end{tabular}

I
I
I
I
I
I
I
I
I
I
I
I
I
I
I
J
J
J
J
J
J
J
K
L
L
L
L

Correlation of Lithologic and Cooling Units with Chemical Types, Sites 482, 483, and 485

\begin{tabular}{|c|c|c|c|c|c|c|}
\hline $\begin{array}{l}\text { Lithologic } \\
\text { Unit }\end{array}$ & $\begin{array}{l}\text { Top } \\
(\mathrm{m})^{\mathrm{a}}\end{array}$ & $\begin{array}{l}\text { Base } \\
(\mathrm{m})^{\mathrm{a}}\end{array}$ & $\begin{array}{c}\text { Cooling } \\
\text { Units }\end{array}$ & $\begin{array}{l}\text { Phenocryst } \\
\text { Assemblage }\end{array}$ & $\begin{array}{l}\text { Core-Section } \\
\text { (level in cm) }\end{array}$ & $\begin{array}{c}\text { Chemical } \\
\text { Type }\end{array}$ \\
\hline \multicolumn{7}{|l|}{ Hole 482B } \\
\hline 1 & 136.5 & 158.0 & $1-4$ & Aphyric & $10-7.8$ to $15-1,115$ & A, F \\
\hline 2 & 158.0 & 174.7 & $10-11$ & Plagioclase & $15-1,115$ to $17-2,150$ & B \\
\hline \multicolumn{7}{|c|}{ Sedimentary intercalation } \\
\hline 3 & 184.1 & 186.2 & 12 & Plagioclase & $18-1,90$ to $18-2,107$ & B \\
\hline 4 & 193.5 & 199.5 & 13 & Plagioclase & $19-1,60$ to $20-2,83$ & C \\
\hline 5 & 199.5 & 201.5 & 14 & Plagioclase-Clinopyroxene & $20-2,83$ to $20-3,130$ & D \\
\hline 6 & 201.5 & 205.0 & 15 & Plagioclase-Clinopyroxene-Olivine & $20-3,130$ to $21-3,50$ & $\mathrm{E}$ \\
\hline 7 & 205.0 & 220.3 & 16 & Plagioclase-Clinopyroxene-Olivine & $21-3,50$ to $23-1,30$ & D \\
\hline \multicolumn{7}{|c|}{ Sedimentary intercalation } \\
\hline 8 & 224.7 & 229.0 & 17 & Aphyric & $24-1,25$ to $24-3,130$ & D \\
\hline \multicolumn{7}{|l|}{ Hole $482 \mathrm{C}$} \\
\hline 1 & 135.5 & 157.0 & $1-2$ & Aphyric & $9-1,60$ to $12-1,0$ & A \\
\hline 2 & 157.0 & 184.0 & $3-7$ & Plagioclase-Clinopyroxene-Olivine & $12-1,0$ to $15-4,145$ & F, B \\
\hline \multicolumn{7}{|l|}{ Hole 482D } \\
\hline 1 & 138.0 & 139.6 & $1-2$ & Aphyric & $8-1,0$ to $8-2,30$ & A \\
\hline \multicolumn{7}{|c|}{ Sedimentary intercalation } \\
\hline 2 & 141.7 & 154.0 & 3 & Aphyric & $9-1,15$ to $10-3,60$ & F. E \\
\hline \multicolumn{7}{|c|}{ Sedimentary intercalation } \\
\hline 3 & 154.1 & 169.6 & $4-10$ & Aphyric & $10-3,65$ to $12-1,130$ & $\mathrm{~F}$ \\
\hline 4 & 169.6 & 186.5 & $11-16$ & Plagioclase & $12-1,130$ to $13-3,35$ & B \\
\hline \multicolumn{7}{|l|}{ Hole 483} \\
\hline 1 & 110.0 & 111.0 & 1 & Plagioclase-Olivine & $13-4,5$ to $13-4,95$ & A \\
\hline \multicolumn{7}{|c|}{ Sedimentary intercalation } \\
\hline 2 & 115.0 & 127.0 & $2(a-b)$ & Aphyric & $14-1,5$ to $15-2,128$ & B \\
\hline \multicolumn{7}{|c|}{ Sedimentary intercalation } \\
\hline 3 & 127.0 & 135.8 & 3 & Aphyric & $15-2,128$ to $16-3,12$ & c \\
\hline \multicolumn{7}{|c|}{ Sedimentary intercalation } \\
\hline 4 & 142.2 & 145.0 & 4 & Aphyric & $17-1,18$ to $17-3,24$ & D \\
\hline \multicolumn{7}{|c|}{ Sedimentary intercalation } \\
\hline 5 & 156.5 & 160.1 & 5 & Aphyric & $18-4,130$ to $19-1,10$ & $\mathrm{E}$ \\
\hline \multicolumn{7}{|c|}{ Sedimentary intercalation } \\
\hline $6 a$ & & $(171.0)$ & & & unrecovered & F? \\
\hline \multicolumn{7}{|c|}{ Sedimentary intercalation } \\
\hline $6 \mathrm{~b}$ & 169.0 & 186.5 & $6-27$ & Plagioclase-Olivine-Clinopyroxene & $20-1,5$ to $22-4,60$ & $\mathrm{~F}$ \\
\hline $6 c$ & 186.5 & 188.5 & 28 & Plagioclase-Olivine & $22-4,60$ to $23-2,20$ & F-X \\
\hline $6 d$ & 188.5 & 190.0 & $29-30$ & Plagioclase-Olivine-Clinopyroxene & $23-2,20$ to $23-2,150$ & $\mathrm{~F}$ \\
\hline
\end{tabular}


M. F. J. FLOWER, R. G. PRITCHARD, H.-U. SCHMINCKE, P. T. ROBINSON

Appendix B. (Continued).

\begin{tabular}{|c|c|c|c|c|c|c|}
\hline $\begin{array}{l}\text { Lithologic } \\
\text { Unit }\end{array}$ & $\begin{array}{l}\text { Top } \\
(\mathrm{m})^{\mathrm{a}}\end{array}$ & $\begin{array}{l}\text { Base } \\
(\mathrm{m})^{\mathrm{a}}\end{array}$ & $\begin{array}{l}\text { Cooling } \\
\text { Units }\end{array}$ & $\begin{array}{l}\text { Phenocryst } \\
\text { Assemblage }\end{array}$ & $\begin{array}{l}\text { Core-Section } \\
\text { (level in } \mathrm{cm} \text { ) }\end{array}$ & $\begin{array}{c}\text { Chemical } \\
\text { Type }\end{array}$ \\
\hline \multicolumn{7}{|c|}{ Sedimentary intercalation } \\
\hline $7 \mathrm{a}$ & 191.5 & 200.3 & $31-34$ & Plagioclase-Olivine & $24-1,5$ to $26-1,40$ & $\mathrm{H}$ \\
\hline \multicolumn{7}{|c|}{ Sedimentary intercalation } \\
\hline $7 \mathrm{~b}$ & 200.4 & 204.5 & 35 & Plagioclase & $26-1,50$ to $26-3,150$ & $\mathrm{H}$ \\
\hline \multicolumn{7}{|l|}{ Hole 483B } \\
\hline 1 & 110.0 & 111.3 & 1 & Plagioclase-Olivine & $2-7,0$ to $3-1,75$ & A \\
\hline \multicolumn{7}{|c|}{ Sedimentary intercalation } \\
\hline \multicolumn{7}{|c|}{ Sedimentary intercalation } \\
\hline $3^{3}$ & 133.0 & 136.4 & 3 & Aphyric & $7-1,7$ to $7-3,75$ & C \\
\hline \multicolumn{7}{|c|}{ Sedimentary intercalation } \\
\hline 4 & 137.6 & 146.8 & 4 & Aphyric & $8-1,10$ to $9-1,44$ & D \\
\hline \multicolumn{7}{|c|}{ Sedimentary intercalation } \\
\hline $5 \mathrm{a}$ & 169.0 & 175.0 & $6-10$ & Plagioclase-Olivine-Clinopyroxene & $12-1,0$ to $13-1,10$ & 1 \\
\hline $5 b$ & 175.0 & 184.6 & $11-22$ & Plagioclase-Olivine-Clinopyroxene & $13-1,10$ to $15-1,6$ & $\mathrm{~F}$ \\
\hline \multicolumn{7}{|c|}{ Sedimentary intercalation } \\
\hline $\begin{array}{c}6 \mathrm{a} \\
\text { Sedimentary }\end{array}$ & 194.0 & 197.3 & 23 & Aphyric & $17-1,0$ to $17-3,45$ & $\mathrm{H}$ \\
\hline \multicolumn{7}{|c|}{ Sedimentary intercalation } \\
\hline $6 \mathrm{~b}$ & 199.0 & 204.6 & 24 & Aphyric & $18-1,0$ to $19-1,65$ & $\mathrm{H}$ \\
\hline \multicolumn{7}{|c|}{ Sedimentary intercalation } \\
\hline $6 c$ & 204.6 & 206.2 & 25 & Aphyric & $19-1,65$ to $19-2,85$ & $\mathrm{H}$ \\
\hline $6 \mathrm{~d}$ & 206.2 & 210.5 & 26 & Plagioclase-Olivine-Clinopyroxene & $19-2,85$ to $20-2,65$ & $\mathrm{H}$ \\
\hline \multicolumn{7}{|c|}{ Sedimentary intercalation } \\
\hline $7 \mathrm{a}$ & 211.1 & 213.0 & 27 & Plagioclașe-Olivine & $20-2,130$ to $20-3,30$ & $\mathrm{H}$ ? \\
\hline $7 \mathrm{~b}$ & 213.0 & 227.7 & $28-56$ & Plagioclase-Clinopyroxene-Olivine & $21-1,0$ to $24-1,145$ & $\mathrm{~J}$ \\
\hline \multicolumn{7}{|c|}{ Sedimentary intercalation } \\
\hline $8 \mathrm{a}$ & 227.7 & 232.4 & 57 & Plagioclase-Clinopyroxene-Olivine & $24-1,145$ to $25-2,5$ & K \\
\hline \multicolumn{7}{|c|}{ Sedimentary intercalation } \\
\hline $8 \mathrm{~b}$ & 232.6 & 236.9 & 58 & Plagioclase-Olivine & $25-2,20$ to $26-1,150$ & K \\
\hline \multicolumn{7}{|c|}{ Sedimentary intercalation } \\
\hline $8 c$ & 237.0 & 249.5 & 59 & Plagioclase-Olivine & $26-2,5$ to $29-1,65$ & $\mathrm{~K}$ \\
\hline \multicolumn{7}{|c|}{ acter } \\
\hline 9 & 249.5 & 267.0 & $60-82$ & Plagioclase-Olivine-Clinopyroxene & $29-1,65$ to $32-3,82$ & L, M \\
\hline \multicolumn{7}{|l|}{ Hole $483 \mathrm{C}$} \\
\hline 1 & 109.5 & 114.0 & 1 & Aphyric & $4-2,47$ to $4-5,120$ & A \\
\hline \multicolumn{7}{|l|}{$485 \mathrm{~A}$} \\
\hline 1 & 153.5 & 159.4 & $1-5$ & Plagioclase-Olivine-Clinopyroxene & $11-3,55$ to $13-1,140$ & $A-E$ \\
\hline \multicolumn{7}{|c|}{ Sedimentary intercalation } \\
\hline$\stackrel{2}{\text { Sedimentary }}$ & 180.5 & 184.0 & ? & Plagioclase & $17-1,0$ to $18-1,58$ & $\mathrm{Z}$ \\
\hline \multicolumn{7}{|c|}{ Sedimentary intercalation } \\
\hline \multicolumn{7}{|c|}{ Sedimentary intercalation } \\
\hline $\begin{array}{c}4 \\
\text { Sedimentary }\end{array}$ & 212.0 & 226.2 & 8 & Plagioclase-Olivine & $23-1,50$ to $26-1,20$ & $\mathrm{H}$ \\
\hline$\underset{5}{\text { Sedimentary }}$ & interca & $\begin{array}{l}\text { ation } \\
270.4\end{array}$ & 9 & & & \\
\hline$\stackrel{5}{\text { Sedimentar }}$ & 239.5 & $\begin{array}{l}270.4 \\
\text { ation }\end{array}$ & & Plagioclase & $29-1,0$ to $33-2,95$ & 1 \\
\hline $\begin{array}{l}\text { Sedimentar } \\
6\end{array}$ & $\begin{array}{l}y \text { interca } \\
277.5\end{array}$ & $\begin{array}{l}\text { ation } \\
294.0\end{array}$ & 10 & Plagioclase-Olivine & $34-1,56$ to $35-6,55$ & J \\
\hline Sedimentary & interca & ation & & & & $J$ \\
\hline 7 & 298.6 & 298.7 & 11 & Plagioclase & $36-3,64$ to $36-c c, 31$ & K \\
\hline Sedimentar & $y$ interca & ation & & & & \\
\hline 8 & 314.5 & 328.5 & 12 & Plagioclase & $38-2,2$ to $39-5,60$ & L \\
\hline
\end{tabular}

a Calculated from core log and corrected for spacers. 